\title{
Research
}

\section{Assessing the Effect of Phenotyping Scoring Systems and SNP Calling and Filtering Parameters on Detection of QTL Associated with Reaction of Brassica napus to Sclerotinia sclerotiorum}

\author{
Fereshteh Shahoveisi ${ }^{1} \mid$ Atena Oladzad $^{2}$ | Luis E. del Río Mendoza ${ }^{1,+}$ (D) Seyedali Hosseinirad $^{2}$ | \\ Susan Ruud ${ }^{1}$ | Bruna Rissato ${ }^{3}$ |
}

${ }^{1}$ Department of Plant Pathology, North Dakota State University, Fargo, ND 58108, U.S.A.

2 Department of Plant Sciences, North Dakota State University, Fargo, ND 58108, U.S.A.

${ }^{3}$ Department of Agronomy, Maringa State University, Maringa, Parana 87020900, Brazil

† Corresponding author: L. E. del Río; luis.delriomendoza@ndsu.edu

Accepted for publication 28 January 2021.

\section{Funding}

United States Department of Agriculture-Agricultural Research Service (USDA-ARS) National Sclerotinia Initiative Non-Assistance Cooperative Agreements numbers 58-3060-6-032 and 58-5442-5-289, and USDA-National Institute of Food and Agriculture Hatch Program Awards ND02349 and ND02240.

$\boldsymbol{e}$-Xtra: Supplementary tables and a supplementary figure are available online.

The author(s) declare no conflict of interest.

\begin{abstract}
The polyploid nature of canola (Brassica napus) represents a challenge for the accurate identification of single-nucleotide polymorphisms (SNPs) and the detection of quantitative trait loci (QTL). In this study, combinations of eight phenotyping scoring systems and six SNP calling and filtering parameters were evaluated for their efficiency in detection of QTL associated with response to Sclerotinia stem rot, caused by Sclerotinia sclerotiorum, in two doubled haploid canola mapping populations. Most QTL were detected in lesion length, relative areas under the disease progress curve (rAUDPC) for lesion length, and binomial-plant mortality data sets. Binomial data derived from lesion size were less efficient in QTL detection. Inclusion of additional phenotypic sets to the analysis increased the numbers of significant QTL by 2.3-fold; however, the continuous data sets were more efficient. Between two filtering parameters used to analyze genotyping-bysequencing data, imputation of missing data increased QTL detection in one population with a high level of missing data but not in the other. Inclusion of segregation-distorted SNPs increased QTL detection but did not impact their $R^{2}$ values significantly. In all, 12 of 16 detected QTL were on chromosomes A02 and C01, and the rest were on A07, A09, and C03. Marker A02-7594120, associated with a QTL on chromosome A02, was detected in both populations. Results of this study suggest that the impact of genotypic variant calling and filtering parameters may be population dependent while deriving additional phenotyping scoring systems such as rAUDPC datasets and mortality binary may improve QTL detection efficiency.
\end{abstract}

Keywords: Brassica napus, doubled haploid population, phenotyping scoring systems, QTL mapping, Sclerotinia sclerotiorum, SNP calling and filtering 
Canola or oilseed rape (Brassica napus L., AACC/ 2n $=4 \mathrm{x}=$ 38 ) is an important allopolyploid oilseed crop characterized for producing oil with less than $2 \%$ erucic acid (Snowdon et al. 2007). The economic value of the crop in the United States was estimated at \$494 million in 2018 (USDA-NASS 2019). The species was formed from spontaneous hybridization events between B. oleracea $(2 \mathrm{n}=2 \mathrm{x}=18, \mathrm{CC})$ and $B$. rapa $(2 \mathrm{n}=2 \mathrm{x}=20, \mathrm{AA})$ approximately 6,700 years ago (Howell et al. 2008; Sun et al. 2017). Breeding programs have had a significant role in shaping the agronomic traits, including disease resistance, exhibited by modern cultivars but, in doing so, they also have reduced their genetic diversity. One of the effective strategies to increase the variation in the genetic basis of the species, including agronomic traits and disease resistance, is introduction of new genetic sources such as wild relatives and plant introduction (PI) lines into the breeding programs (Amosova et al. 2019; Delourme et al. 2013; Girke et al. 2012).

Sclerotinia stem rot (SSR) caused by Sclerotinia sclerotiorum is one of the major fungal diseases of canola (B. napus L.) worldwide (Saharan and Mehta 2008). In North Dakota, every unit increase in SSR incidence reduces canola yield by $0.5 \%$ (del Río et al. 2007). Several strategies such as rotation with nonhost crops, using forecasting models as a decision tool, fungicide applications, and planting resistant varieties are recommended for SSR management (Derbyshire and Denton-Giles 2016; Rothmann and McLaren 2018; Shahoveisi and del Río Mendoza 2020).

Breeding for resistant cultivars is a nature-friendly, economic, and sustainable approach to reduce the severity of SSR. However, breeding for quantitative traits such as resistance to $S$. sclerotiorum (Disi et al. 2014; Wu et al. 2019) is challenging, and limited progress has been made in canola. For example, within the past 10 years, almost twice as many quantitative trait loci (QTL) have been identified in dry bean (Phaseolus vulgaris L.) than in canola (Arkwazee 2018; Lara et al. 2014; Miklas et al. 2013; Mkwaila et al. 2011; Pérez-Vega et al. 2012; Qasim et al. 2020; Soule et al. 2011; Vasconcellos et al. 2017; Wei et al. 2014; Wu et al. 2013, 2019; Yin et al. 2010; Zhao and Meng 2003; Zhao et al. 2006). Nevertheless, QTL associated with SSR in canola have been identified on 16 of its 19 chromosomes (e.g., A01, A02, A03, A06, A07, A08, A09, A10, C01, C02, C03, C04, C05, C06, C07, and C08) (Gyawali et al. 2016; Qasim et al. 2020; Wei et al. 2014; Wu et al. 2019; Zhao and Meng 2003; Zhao et al. 2006). Despite these efforts, however, most cultivars remain susceptible to the disease; therefore, additional work is required to identify QTL that could facilitate transfer of resistance to SSR into modern canola cultivars.

Detection of QTL requires the development of phenotypic and genotypic data from individuals in a mapping population. To generate phenotypic data, S. sclerotiorum is commonly inoculated on canola plants using agar plug stem, toothpick, and detached-leaf methods (Arahana et al. 2001; Garg et al. 2013; Wu et al. 2013, 2019; Zhao and Meng 2003; Zhao et al. 2006). Among these methods, agar plug provides the closest semblance to natural infection in the field because the inoculum is deposited on the surface of the plant rather than inside (toothpick method) and the plant parts are not excised (detached leaf). In terms of plant response quantification, average lesion length and days to wilt at single points in time are the most reported methods in QTL mapping studies (Derbyshire and Denton-Giles 2016). These two variables offer a snapshot of the reaction of plants to infection that, in some instances, may not be accurate enough. In contrast, the area under the disease progress curve (AUDPC) provides a more complete picture of that reaction; however, its use in QTL studies has been limited and not thoroughly investigated. More recently, Oladzad et al. (2019) and Zitnick-Anderson et al. (2020), working with diploid crops, showed that reducing the number of categorical disease severities from nine to three (e.g., resistant, moderately resistant, and susceptible) (polynomial distribution) or just two (e.g., resistant and susceptible) (binomial distribution) may increase the power of detecting QTL associated with reaction to root pathogens. Given the importance of phenotyping data in detecting and mapping disease resistance QTL, a comprehensive study in which a variety of phenotyping systems are investigated will provide valuable insights into plant pathology genome studies.

The methods used to generate genotypic data have improved markedly in recent years. Genotyping-by-sequencing (GBS) and single-nucleotide polymorphism (SNP) arrays are the most common and efficient genotyping methods used in recent QTL mapping studies for B. napus (ArifUzZaman et al. 2016; Wu et al. 2019; Yu et al. 2017). The data produced by these platforms, however, need to be processed using variant calling and filtering methods before being used for QTL analyses. Imputation of missing data is a common process in SNP pipeline development for genome-wide association studies (GWAS) (Lu et al. 2019; Oladzad et al. 2019; Porcu et al. 2013). This procedure can be beneficial when low-coverage GBS data are used to genotype the mapping population (Zheng et al. 2018) because it may increase the power of the QTL mapping and marker density (Porcu et al. 2013). Data imputation is used less frequently in biparental QTL mapping studies where it could be beneficial for populations with high number of missing data and heterozygous calls. Despite the availability of several promising imputation algorithms developed for recombinant inbred line (Xie et al. 2010), full-sib (Swarts et al. 2014), and $F_{2}$ and $F_{1} B C$ (Fragoso et al. 2016) mapping populations, researchers have yet to answer the question of whether imputation increases the QTL detection power in biparental population studies. Distortion from Mendelian segregation law is a common phenomenon in biparental mapping populations (Xu 2008). Deviation from Mendelian segregation could be determined using a $\chi^{2}$ test (Dai et al. 2017; Nixon 2006). However, the benefits of discarding markers with distorted segregation are still debatable and researchers may discard these types of markers in the QTL analysis (ArifUzZaman et al. 2016; da Silva et al. 2019; Huang and Yan 2019; Pascual et al. 2016) or keep them (Tyrka et al. 2018). Retaining markers with distorted segregation may result in better coverage of linkage groups and improve the output of the QTL mapping (Zuo et al. 2019). One of the goals of the current study was to compare the results of the QTL analyses of doubled haploid (DH) populations in the presence and absence of segregation distortion loci.

Identification of QTL associated with disease resistance is needed for implementation of marker-assisted selection in breeding programs. Despite its importance, however, research that compares the efficacy of different phenotyping scoring system is lacking. Revealing the effect of scoring systems on detecting QTL associated with disease resistance could assist researchers to avoid excessive phenotyping work and speed up the accurate evaluation of a phenotype; furthermore, deriving and using multiple phenotyping datasets might result in detection of additional QTL. In this study, the impact of using multiple continuous and categorical phenotyping scoring systems in combination with SNP calling and filtering parameters to detect QTL associated with reaction of $B$. napus to $S$. sclerotiorum was evaluated. 


\section{MATERIALS AND METHODS}

\section{DH population development}

The first DH population, T63DH, with 127 individuals, was constructed from a cross between Topas and Nep63. Topas is an open-pollinated spring cultivar with a very low level of SSR resistance which is amenable for microspore culture process; therefore, it was used as the female in the crosses. Nep63 is a semiwinter DH line produced from a cross between PI 458940 and PI 649136 of the U.S. National Plant Germplasm System, which were originally collected from Japan and Germany, respectively. Nep63 is highly resistant to SSR and it was used as the male in the crosses. The second mapping population, T54DH, with 201 lines, was generated from a cross between Topas and PI 436554, which is a spring type line originated from China.

For DH production, a modified microspore culture process reported by Ferrie et al. (2005) was used. Approximately 150 unopened floral buds, 2 to $3 \mathrm{~mm}$ long, were collected from $\mathrm{F}_{1}$ plants of each cross. Buds were surface disinfested in an aqueous solution of $\mathrm{NaOCl}$ at $0.525 \%$ ( $\mathrm{vol} / \mathrm{vol}$ ) for $10 \mathrm{~min}$ and rinsed three times with cold, distilled, autoclaved water. Microspores were released from the buds by grinding them in $5 \mathrm{ml}$ of half-strength B5 medium (Gamborg et al. 1968) with $13 \%$ sucrose (1/2 B5-13). The microspores were filtered and suspended in $15 \mathrm{ml}$ of clean 1/2 B5-13 medium and centrifuged for $3 \mathrm{~min}$ at $800 \mathrm{rpm}$, after which the supernatant was carefully discarded. The pelleted microspores were resuspended in $5 \mathrm{ml}$ of clean 1/2B5-13 medium. This process was repeated twice and, after the third centrifuge, the pellet was suspended in $5 \mathrm{ml}$ of NLN-13 medium (PhytoTechnologies Labs, Lenexa, KS, U.S.A.) and the concentration of the microspores was adjusted to $10^{5}$ microspores $/ \mathrm{ml}$. The suspension was poured into deep Petri dishes $(20 \mathrm{ml} / \mathrm{dish})$ and incubated in the following conditions: at $32^{\circ} \mathrm{C}$ and dark conditions for 3 days, then at $24^{\circ} \mathrm{C}$ for 3 weeks, and, finally, on a shaker at 22 to $24^{\circ} \mathrm{C}$ (70 rpm and $24 \mathrm{~h}$ of light) until embryos reached 2 to $3 \mathrm{~mm}$ in length. Subsequently, $50 \mu \mathrm{M}$ colchicine (MilliporeSigma, Burlington, MA, U.S.A.) solution was added to embryos for $72 \mathrm{~h}$ at $24^{\circ} \mathrm{C}$. After this period, embryos were transferred into solid B5 medium (MilliporeSigma). Germinated plantlets were transferred to 5 -in. $(12.7-\mathrm{cm})$ pots in greenhouse. The size and density of leaf stomata from DH plantlets were compared with that of a haploid check to verify the production of DH plants (Mohammadi et al. 2012). At flowering, plants were covered with pollination bags to avoid cross pollination. Fertile DH plants in each population along with their parental lines were used for phenotyping and genotyping.

\section{Phenotyping}

Greenhouse trials were conducted to assess the reaction of both populations against $S$. sclerotiorum. All of the $\mathrm{DH}$ lines and parental lines were inoculated 45 to 55 days after planting using an agar stem method. The experiment was conducted twice in randomized complete blocks with five replications in each trial. Agar plugs, $6 \mathrm{~mm}$ in diameter, containing hyphal tips of isolate WM031 were made from 2-day-old S. sclerotiorum colonies growing on potato dextrose agar medium. Isolate WM031 was originally collected from Nebraska, United States and is considered highly virulent on canola. Single plugs were placed with the mycelium side in contact with the main stem of plants between the third and fourth leaves. The plugs were secured in position by wrapping them to the stems with a piece of Parafilm. The length of the lesions and plant mortality were measured starting 3 days postinoculation (dpi) and then at 2-day intervals until $11 \mathrm{dpi}$. Plants were considered dead when irreversible wilting symptoms were observed on plant parts above the inoculated area of the stem. To characterize the response of each population to the disease, their average rates of stem lesion expansion and mortality were calculated using linear and nonlinear regressions.

\section{Phenotyping scoring systems}

The phenotyping scoring systems were as follows.

Lesion, binomial-lesion, and polynomial-lesion lengths. The average stem lesion-length data collected at 9 dpi were used to derive binomial and polynomial sets. For the binomial dataset, the lines were given a value of 0 if their average lesion length was $\leq 3 \mathrm{~cm}$. These lines were considered resistant. The remaining lines were considered susceptible and were assigned a value of 1 . For the polynomial dataset, resistant lines from the binomial set remain as resistant and were assigned a value of 1 while the susceptible lines were further split into intermediate and susceptible categories, with the latter group having lesions $>6 \mathrm{~cm}$ in length. Intermediate lines were assigned a value of 2 and susceptible lines a value of 3 (Supplementary Table S1).

Relative AUDPC (rAUDPC). Stem lesion length (in centimeters), was used to calculate three rAUDPC datasets for each line. For rAUDPC1, data from all reading times were used; for rAUDPC2, data from 5th to $11 \mathrm{dpi}$ were used; and for rAUDPC 3, data from 3, 7, and 11 dpi were used. Because lesion length was not measured once a plant was declared dead, the largest lesion length at each reading time in every replication was identified and ascribed to dead plants. In this way, we ensured that each rAUDPC would be larger on susceptible lines than on resistant lines. At the same time, each susceptible line with greater plant mortality would have larger rAUDPC than susceptible plants with lower mortality. The rAUDPC was calculated as follows:

$$
r A U D P C=\frac{\sum\left(\frac{y_{i}+y_{i+1}}{2}\right)\left(t_{i+1}-t_{i}\right)}{n}
$$

where $y_{i}$ and $y_{i+1}$ represent lesion lengths at two consecutive reading times, $t_{i}$ and $t_{i+1}$ represent the time in days postinoculation for the two readings, and $n$ represents the number of days between the first and last reading times.

Binomial and polynomial plant mortality. Data from plant mortality calculated at 11 dpi was used to create binomial and polynomial datasets. For the binomial set, lines with plant mortality $\leq 20 \%$ were classified as resistant and assigned a value of 0 ; the remaining lines were considered susceptible and assigned a value of 1 . For the polynomial dataset, lines considered resistant in the binomial dataset were classified as resistant and assigned a value of 1 while susceptible lines were split into moderately resistant and susceptible, with the latter group having plant mortality $>60 \%$. Intermediate lines were assigned a value of 2 and susceptible lines a value of 3 (Supplementary Table S1).

Datasets for lesion length and rAUDPC were tested for normality ( $\alpha=0.05)$ using the Univariate procedure of SAS (version 9.4; SAS Institute, Cary, NC, U.S.A.). Homogeneity of variances ( $\alpha=0.05$ ) for each population were tested by using Levene's test. When permitted, trials were combined, and average value of each line was calculated across trials and replications. 


\section{Genotyping}

DNA extraction and sequencing. Leaf samples were collected from 20-day-old plants and DNA was extracted with Qiagen DNeasy 96 Plant kit (Qiagen, Hilden, Germany) for the T54DH population and with MagMax Plant DNA Isolation kit (Thermo Fisher Scientific, Walthman, MA, U.S.A.) for the T63DH population. The decision to switch DNA extraction kits was based on the simplicity of working with the MagMax kit. In total, 332 DNA samples, 127 progeny lines from the T63DH population, and 201 from the T54DH population and the four parents, were sent to University of Minnesota Genomics center (Minneapolis, MN, U.S.A.) for sequencing analyses. GBS dual-index libraries were created using ApeK1 enzyme (Elshire et al. 2011) and were combined into a single pool and sequenced, 100-bp single end, across two lanes of a NovaSeq SP. Subsequently, sequencing barcodes and adaptors were removed.

SNP calling. Sequencing reads were trimmed for a minimum quality score of 20 and minimum sequence length of $80 \mathrm{bp}$ using Sickle (Joshi and Fass 2011). The reads were then mapped against B. napus reference genome, Darmor-bzh v4.1 (http:// www.genoscope.cns.fr/brassicanapus/data/) (Chalhoub et al. 2014), using Burrows-Wheeler Alignment Tool's Maximal Exact Match algorithm (Li 2013) to generate binary alignment map (BAM) files. SNP calling was conducted using following pipelines (SNP data are presented in Supplementary Table S2).

Nonimputed data. The BAM files were sorted and indexed using SAMtools ( $\mathrm{Li}$ et al. 2009) for each population. Using VarScan v2.3.7 (Koboldt et al. 2012), SNPs were called using SAMtools mpileup output files. The minimum coverage and minimum read numbers were set at three and two, respectively. The SNPs and genotypes were removed if (i) the reference allele was N, (ii) $20 \%$ of the SNPs were not called at the given position, (iii) a genotype had more than $40 \%$ missing SNPs, and (iv) a genotype had more than $30 \%$ heterozygosity. Only markers that were polymorphic between parents and had homozygous alleles were used for the construction of the linkage groups.

Imputed data. Similar filtering and SNP calling criteria were used for this pipeline but only reads with high mapping quality (MAPQ 10) were extracted from the BAM files using SAMtools. Subsequently, the SNPs with less than $20 \%$ missing data were imputed using fastPHASE (Scheet and Stephens 2006). After imputation, the genotypes were tested for heterozygosity level at threshold of $30 \%$. Polymorphic markers with homozygous alleles were used for construction of the genetic maps.

\section{Linkage map construction and QTL analyses}

MapDisto software (Lorieux 2012) was used to construct genetic linkage maps. Two additional versions of each map were prepared by filtering out SNPs that showed significant departures from the expected Mendelian segregation using a $\chi^{2}$ test with $\alpha$ of 0.001 and 0.0001 . Recombination frequency was measured using the Kosambi mapping function (Kosambi 1943) and sum of adjacent recombination frequencies criteria were used for ordering the loci for all nine genotyping datasets. Initially, linkage groups were generated at a recombination frequency of 0.3 and a stringent logarithm of odds (LOD) value of 10 for datasets with the $\chi^{2}$ test and 20 for those datasets on which this test was not conducted. The final framework map for each linkage group was created at LOD $=4$ for all of the datasets $\left(\chi^{2}\right.$ tests were conducted at this step). Reversed groups were flipped using the "flip group" option. QTL associated with SSR response were identified using QTL IciMapping 4.2 (Meng et al. 2015) using its inclusive composite interval mapping algorithm, which increases the detection power and reduces false detection. The walking step was set to 1 centimorgan and a 1,000-permutations test at a significant level of $P<0.05$; the percentage of phenotypic variation explained by each QTL $\left(R^{2}\right)$ was also determined. Mapchart software version 2.23 was used for the graphical presentation of linkage groups (Voorrips 2002).

\section{Prediction accuracy}

Prediction accuracy of markers linked to the detected QTL in multiple genotyping and phenotyping analyses was calculated. The accuracy of these markers was expressed as the percentages of correct association of the A allele (from the resistant parent) to the resistant phenotypic reaction and $\mathrm{B}$ allele (from the susceptible parent) to the susceptible reaction (OladzadAbbasabadi et al. 2018; Salsman et al. 2018). The accuracy of prediction was evaluated using lesion-length data with imputed (T54DH) or nonimputed (T63DH) genotyping datasets. Lines with lesion lengths smaller than $3 \mathrm{~cm}$ were considered resistant.

\section{RESULTS}

\section{Phenotyping}

Lesion-length and rAUDPC phenotyping datasets were normally distributed $(P>0.11)$ for T63DH and T54DH populations and variances of the trials within each population were homogeneous $(P>0.3)$. Therefore, average values across all trials and replications of each population were calculated for these scoring systems. Length of stem lesions increased almost linearly with time in both populations at an average rate of 0.5 and $0.6 \mathrm{~cm} /$ day in T63DH and T54DH, respectively (Supplementary Fig. S1). In contrast, plant mortality behaved markedly differently. In $\mathrm{T} 54 \mathrm{DH}$, it increased linearly over time at a rate of $0.6 \%$ per day to an average maximum of $6 \%$ whereas, in $\mathrm{T} 63 \mathrm{DH}$, it followed a quadratic function and reached a maximum of $41 \%$ (Supplementary Fig. S1). Because lesion length was not recorded once a plant was declared dead, data on lesion length measured at 9 dpi was used to derive binomial and polynomial datasets. Many lines had a very reduced number of plants alive at $11 \mathrm{dpi}$; however, it provided the most representative dataset for mortality analysis. This indicates that the optimum time for recoding the disease reaction varies depending on the scoring system.

\section{T63DH population}

A range of resistant to susceptible reactions was observed 9 dpi in the population. Approximately $18 \%$ of the population showed an average lesion size of $\leq 3 \mathrm{~cm}$ (resistance reaction), $65 \%$ had lesion length between 3 and $6 \mathrm{~cm}$ (intermediate reaction), and the remaining $17 \%$ were susceptible, with a lesion size $>6 \mathrm{~cm}$. The resistant and susceptible parents had average lesion lengths of 1.5 and $8.2 \mathrm{~cm}$, respectively (Fig. 1A; Table 1). The lesion size of the most susceptible line in this population was, on average, $9 \mathrm{~cm}$, while the most resistant one had a lesion length of $1.2 \mathrm{~cm}$. In the lesion-binomial dataset, $23 \mathrm{DH}$ lines of the population and the resistant parental line, Nep63, were categorized as resistant and $104 \mathrm{DH}$ lines along with the susceptible parent, Topas, as susceptible. In the lesion-polynomial dataset, $23 \mathrm{DH}$ lines and Nep63 were considered resistant, 82 lines showed intermediate reactions, and the remaining 22 lines and Topas were susceptible. 
Using rAUDPC datasets, distribution of the populations was similar in rAUDPC1 and rAUDPC3. Cutoff value to separate resistant and susceptible reactions in rAUDPC datasets was set at $2.5 \mathrm{~cm}$ because that value was associated with a line showing a $3-\mathrm{cm}$ lesion in T63DH. In total, 29, 21, and 32 lines showed resistant reactions in rAUDPC sets 1,2 , and 3 , respectively (Figs. 1B to D). These values represented 22,17 , and $25 \%$ of that population, respectively. The range of rAUDPC values for the resistant parent, Nep63, was 1.4 to $1.5 \mathrm{~cm}$ and for the susceptible parent, Topas, was 5.9 to $7 \mathrm{~cm}$. Plant mortality in the population was observed starting 5 dpi (Supplementary Fig. S1). In the mortality-binomial dataset, 39 lines and the resistant parent had $<20 \%$ plant mortality and 88 lines along with the susceptible parent showed a susceptible reaction with $>20 \%$ plant mortality. In the polynomial dataset, 39 lines were considered resistant, 63 intermediate, and 25 susceptible.

The five most resistant lines in this population had lesion lengths ranging between 1.2 and $1.9 \mathrm{~cm}$ and rAUDPC values between 1.2 and 1.6, 1.2 and 1.8, and 1.1 and $1.6 \mathrm{~cm}$ for rAUDPC1, rAUDPC2, and rAUDPC3, respectively. These lines did not have dead plants.

\section{T54DH population}

This mapping population was, in general, more susceptible to $S$. sclerotiorum than the one derived from Nep63. At 9 dpi, approximately $15 \%$ of lines had average lesion lengths $<3 \mathrm{~cm}, 46 \%$ of its members had lesions between 3 and $6 \mathrm{~cm}$, and the remaining $39 \%$
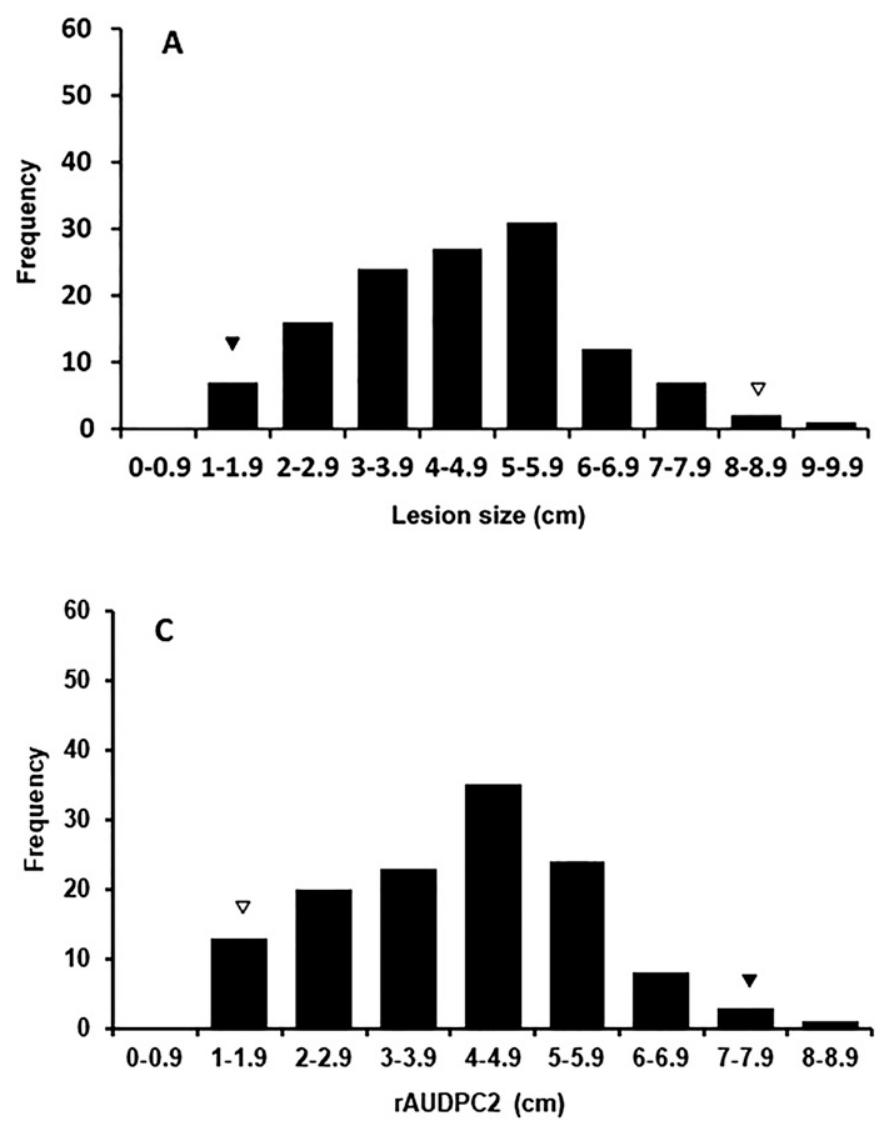

of lines had lesions $>6 \mathrm{~cm}$ at $9 \mathrm{dpi}$ (Fig. 2A; Table 1). At that time, the resistant and susceptible parents had average lesion lengths of 2.5 and $8.5 \mathrm{~cm}$, respectively (Fig. 2A; Table 1). The most susceptible line of this population had, on average, 11.8-cm-long lesions while the most resistant had 1.1-cm-long lesions. In the lesionbinomial dataset, $31 \mathrm{DH}$ lines of the population and the resistant parental line, PI 436554, were categorized as resistant and $170 \mathrm{DH}$ lines along with the parental line, Topas, as susceptible. In the lesion-polynomial dataset, $31 \mathrm{DH}$ lines and Nep63 were considered resistant, 92 lines showed intermediate reactions, and the remaining 78 lines and parental line Topas were susceptible. The rAUDPC cutoff values for resistant and susceptible reactions in T54DH were 3.1, 2.6, and 4.2 for rAUDPC1, rAUDPC2, and rAUDPC3, respectively. The three datasets contained 13, 15, and $12 \%$ resistant lines, respectively. The range of rAUDPC values for the resistant parent, Nep63, was 2.3 to $4.2 \mathrm{~cm}$ and for the susceptible parent, Topas, was 6.1 to $7.1 \mathrm{~cm}$ (Figs. 2B to D). Like the T63DH population, plant mortality was initially observed at $5 \mathrm{dpi}$. For the mortality-binomial dataset, 56 lines and the resistant parent had $<20 \%$ plant mortality and 145 lines along with the susceptible parent showed a susceptible reaction with $>20 \%$ mortality. In the polynomial dataset, 56 lines were considered resistant, 107 intermediate, and 38 susceptible.

The five most resistant lines in this population had lesion lengths ranging between 1.1 and $1.4 \mathrm{~cm}$, and rAUDPC values ranging between 2.1 and 2.6, 1.0 and 1.4 , and 3.2 and $3.8 \mathrm{~cm}$ for rAUDPC1, rAUDPC2, and rAUDPC3, respectively. All five genotypes had survival rates of $100 \%$.
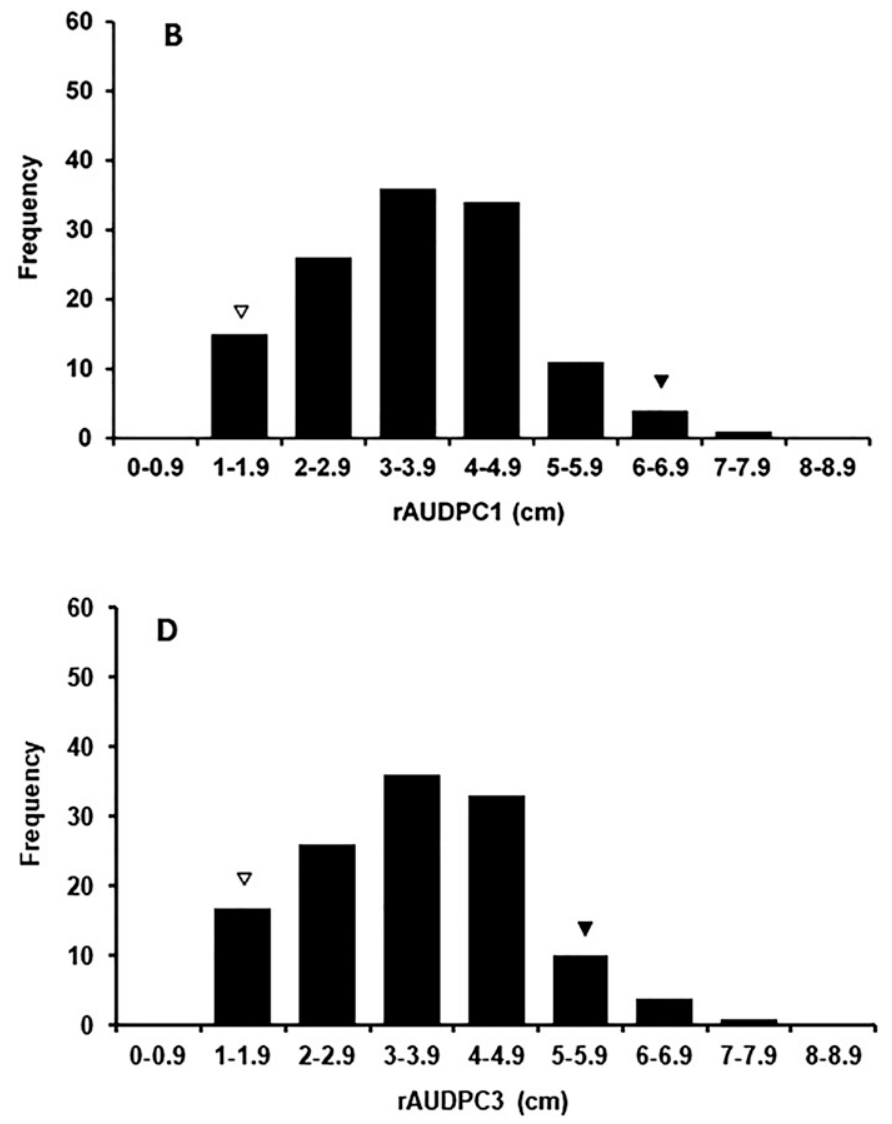

FIGURE 1

Frequency distribution of Brassica napus T63DH population with 127 doubled haploid lines. The location of the resistant (Nep63) and susceptible (Topas) parents are shown by white and black triangles, respectively. A, Lesion-length data set, 9 days postinoculation (dpi); $\mathbf{B}$, relative area under the disease progress curve set 1 (rAUDPC1), calculated using data from 3, 5, 7, 9, and 11 dpi; C, rAUDPC2, calculated using data from 5, 7, 9, and $11 \mathrm{dpi}$; and D, rAUDPC3, calculated using data from 3, 7, and $11 \mathrm{dpi}$. 


\section{Genotyping}

SNP calling. More than 750 million reads were generated for the whole sequencing run and the average yield was approximately 2.4 million reads per library (332 libraries). The mean

\section{TABLE 1}

Phenotypic variation in the response of T54DH and T63DH populations and their parental lines to stem inoculation with Sclerotinia sclerotiorum measured 9 and 11 days postinoculation (dpi) for lesion size and plant mortality, respectively

\begin{tabular}{|c|c|c|c|c|c|}
\hline \multirow{2}{*}{$\begin{array}{l}\text { Population, } \\
\text { scoring system }\end{array}$} & \multicolumn{2}{|c|}{ Parental lines } & \multicolumn{3}{|c|}{ DH population $^{\text {b }}$} \\
\hline & Resistant & Susceptible & Mean & Minimum & Maximum \\
\hline \multicolumn{6}{|l|}{ T63DH } \\
\hline Lesion $(\mathrm{cm})$ & 1.5 & 8.2 & 4.7 & 1.2 & 9 \\
\hline rAUDPC1 (cm) & 1.4 & 6.1 & 3.6 & 1.2 & 7.8 \\
\hline rAUDPC2 $(\mathrm{cm})$ & 1.5 & 7 & 4.1 & 1.2 & 8.7 \\
\hline rAUDPC3 $(\mathrm{cm})$ & 1.5 & 5.9 & 3.6 & 1.1 & 7.6 \\
\hline Mortality (\%) & 10 & 90 & 40 & 0 & 100 \\
\hline \multicolumn{6}{|l|}{$\mathrm{T} 54 \mathrm{DH}$} \\
\hline Lesion (cm) & 2.5 & 8.5 & 5.4 & 1.1 & 11.8 \\
\hline rAUDPC1 $(\mathrm{cm})$ & 3.1 & 6.2 & 4.7 & 2.1 & 8.7 \\
\hline rAUDPC2 $(\mathrm{cm})$ & 2.3 & 6.1 & 4.2 & 1.0 & 8.9 \\
\hline rAUDPC3 $(\mathrm{cm})$ & 4.2 & 7.1 & 5.6 & 3.2 & 9 \\
\hline Mortality (\%) & 10 & 88 & 41.8 & 0 & 100 \\
\hline
\end{tabular}

${ }^{a}$ Relative area under the disease progress curve (rAUDPC) sets 1 to 3 for lesion length with readings between 3 and 11 dpi (rAUDPC1); 5 and 11 dpi (rAUDPC2); and 3, 7, and 11 dpi (rAUDPC3).

${ }^{\mathrm{b}} \mathrm{DH}=$ doubled haploid.
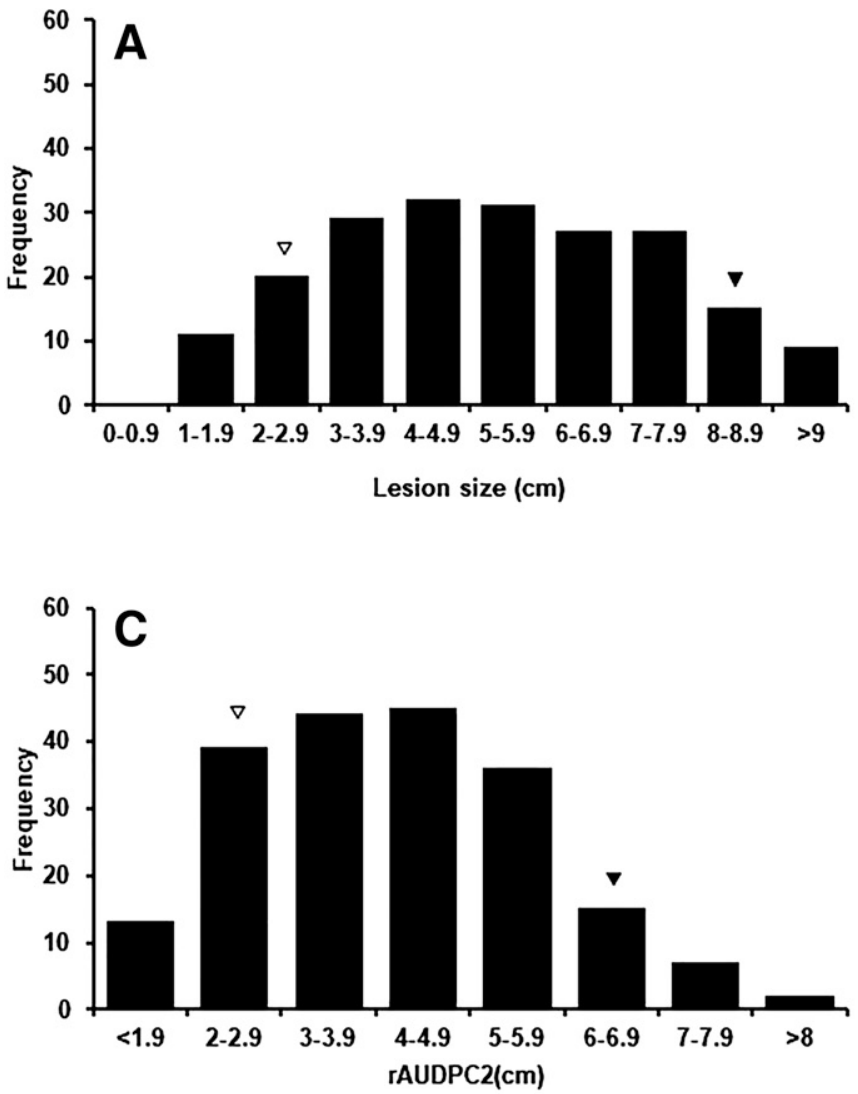

quality score was greater than Q30 for all libraries. In total, $312,641,414$ and 493,048,370 raw reads were generated for $\mathrm{T} 63 \mathrm{DH}$ and $\mathrm{T} 54 \mathrm{DH}$, respectively, and used for further processing for SNP calling.

The impact of the different SNP calling and filtering parameters on the number of markers ascribed to linkage groups varied between populations (Table 2). Imputing the missing data increased the number of markers available for mapping in both populations but with different magnitudes. The number of markers available for mapping in the set of imputed data were 964 for the T63DH population and 1,107 for the T54DH population. The sets with imputed data had 1.5 times more SNPs available for mapping than the nonimputed data in both populations (Table 2). Similarly, the percentage of average markers ascribed to linkage groups in the imputed data was greater in both populations compared with the percentages in the nonimputed set (e.g., 41 and 72\% increase for T63DH and T54DH populations, respectively).

Screening potential SNPs for departures from the 1:1 ratio using the $\chi^{2}$ test for segregation distortion in general reduced the percentage of available markers that were mapped to linkage groups; however, the magnitude of the impact varied with population. The percentage of available markers mapped to linkage groups was 85 and $95 \%$ for the nonimputed and imputed datasets, respectively. When the test was conducted, the average percentage of available markers mapped to linkage groups was reduced by $15 \%$. On $\mathrm{T} 54 \mathrm{DH}$, the percentage of available markers mapped to linkage groups was 17 and 54 for sets with no imputation and imputation, respectively. When the test was
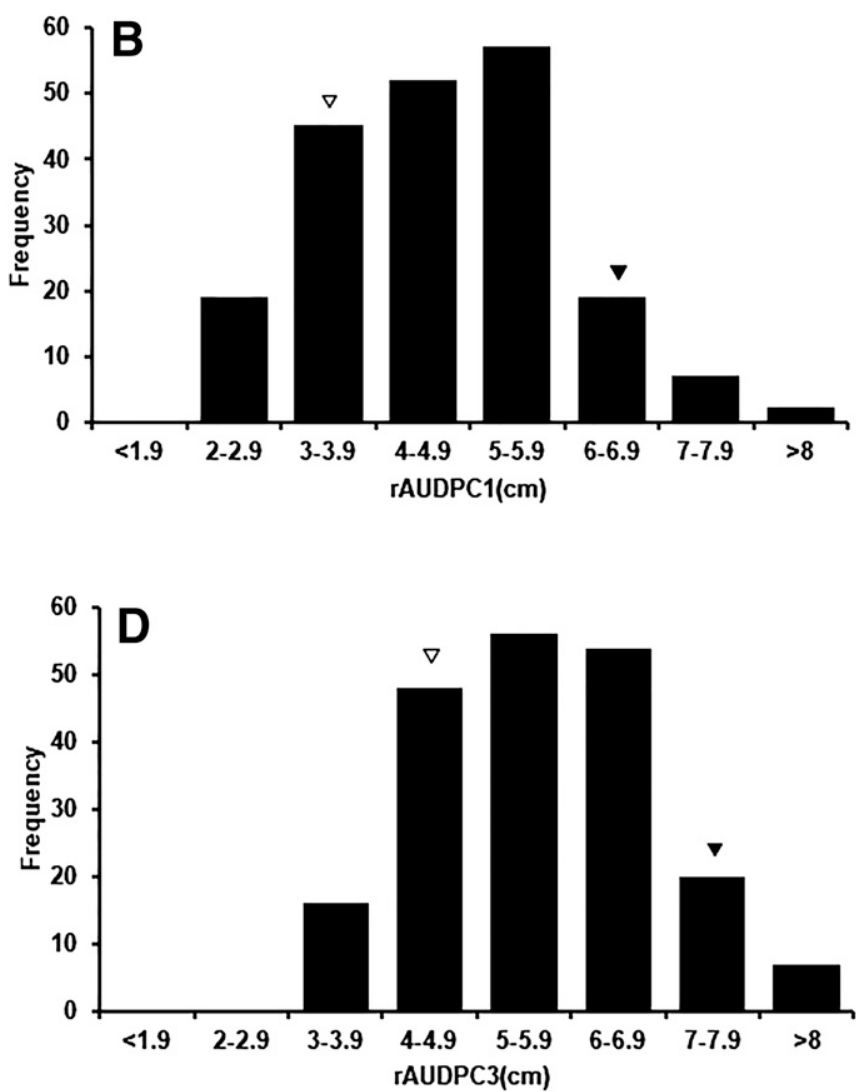

FIGURE 2

Frequency distribution of Brassica napus T54DH population with 201 doubled haploid lines. The mean values of resistant parent (PI436554) and susceptible parent (Topas) are shown by white and black triangles, respectively. A, Lesion-length dataset, 9 days postinoculation (dpi); B, relative area under the disease progress curve set 1 (rAUDPC1), calculated using data from 3, 5, 7, 9, and 11 dpi; C, rAUDPC2, calculated using data from 5, 7, 9, and $11 \mathrm{dpi}$; D, rAUDPC3, calculated using data from 3, 7, and 11 dpi. 
conducted, the average percentage of available markers mapped to linkage groups was reduced by $45 \%$.

The stringency of the $\chi^{2}$ test for segregation distortion, whether using $\alpha$ values of 0.001 or 0.0001 , had a reduced effect on the percentage of available markers mapped to linkage groups from any dataset within both populations (Table 2). On T63DH, conducting the $\chi^{2}$ test using $\alpha=0.001$ resulted in sets with 63 and $79 \%$ of available markers being mapped to linkage groups in nonimputed and imputed datasets, respectively. When the test was conducted using $\alpha=0.0001$, the percentage of markers in T63DH increased between 6 and $12 \%$ in all sets whereas, in T54DH, it increased approximately $2 \%$.

The general order of the markers on linkage maps constructed using different parameters agreed with each other and the reference genome. The complete linkage groups are presented in Supplementary Table S3.

\section{QTL mapping}

The impact of the SNP calling parameters on the number of QTL associated with reaction to disease varied with the population. In $\mathrm{T} 63 \mathrm{DH}$, the use of imputed data with or without a test for segregation distortion resulted in lower numbers of QTL whereas, in T54DH, this approach yielded higher numbers (Table 2). In the latter population, no QTL were detected using nonimputed data. In general, the use of a segregation distortion test did not have a consistent influence on the number and effect $\left(R^{2}\right)$ of QTL detected independent of the population evaluated.

The choice of phenotypic scoring system had a strong impact on the number of QTL detected but that effect varied with population. The original lesion-length set and the rAUDPCs derived from it were the most consistent in allowing detection of significant QTL with high prediction accuracy in both

TABLE 2

Effect of variant calling methods and filtering parameters on the number of single nucleotide polymorphisms (SNPs) available for mapping in two doubled haploid (DH) Brassica napus mapping populations ${ }^{\mathrm{a}}$

\begin{tabular}{lccc}
\hline $\begin{array}{c}\text { Population, SNP } \\
\text { calling, and } \chi^{2} \text { tests }\end{array}$ & $\begin{array}{c}\text { Markers on } \\
\text { LGs/number of LGs }\end{array}$ & $\begin{array}{c}\text { Map length } \\
\text { (centimorgans) }\end{array}$ & QTL \\
\hline T63DH & & & \\
Nonimputed data & & & \\
$\chi^{2} \alpha=0.001$ & $413 / 15$ & 766.87 & 4 \\
$\chi^{2} \alpha=0.0001$ & $494 / 15$ & 822.06 & 5 \\
No $\chi^{2}$ & $562 / 18$ & 951.82 & 4 \\
Imputed data & & & \\
$\chi^{2} \alpha=0.001$ & $759 / 19$ & $1,720.71$ & 1 \\
$\chi^{2} \alpha=0.0001$ & $818 / 20$ & $1,780.49$ & 2 \\
No $\chi^{2}$ & $915 / 29$ & $2,228.62$ & 2 \\
T54DH & & & \\
Nonimputed data & & & \\
$\chi^{2} \alpha=0.001$ & $77 / 8$ & 337 & 0 \\
$\chi^{2} \alpha=0.0001$ & $87 / 8$ & 388.95 & 0 \\
No $\chi^{2}$ & $129 / 17$ & 494.68 & 0 \\
Imputed data & & & \\
$\alpha=0.001$ & $216 / 16$ & $1,220.90$ & 2 \\
$\alpha=0.0001$ & $244 / 17$ & $1,383.74$ & 3 \\
No $\chi^{2}$ & $595 / 27$ & $3,315.47$ & 3 \\
\hline
\end{tabular}

\footnotetext{
${ }^{\mathrm{a}} \mathrm{LG}=$ linkage group and $\mathrm{QTL}=$ quantitative trait loci.
}

${ }^{\mathrm{b}}$ For T63DH, nonimputed data had $126 \mathrm{DH}$ lines and 658 markers available for LG construction, and imputed data had $127 \mathrm{DH}$ lines and 964 markers available for LG construction.

${ }^{c}$ For T54DH, nonimputed data had 200 DH lines and 741 markers available for LG construction, and imputed data had $201 \mathrm{DH}$ lines and 1,107 markers available for LG construction. populations. In $\mathrm{T} 63 \mathrm{DH}$, using the polynomial set derived from lesion length, two QTL were detected, while a single QTL was detected using the derived binomial set. In contrast, the binomial dataset derived from plant mortality yielded six QTL and mortality-polynomial identified only one QTL. Of the 11 QTL detected in this population, some showed overlaps in physical positions; however, we identified them as different QTL to enable the comparison of the methods. In all, 4 of the 11 QTL were detected in each of the three rAUDPC sets and the original lesion-length set, 1 QTL was identified only with lesion length, and the remaining 6 were identified by the mortalitybinomial and polynomial datasets (Table 3). In contrast, in T54DH, four of the five detected QTL were identified using either rAUDPC sets 1, 2 or 3, and the original set for lesion length (Table 4) and a single QTL was detected in the lesionbinomial set. One QTL, with the highest prediction accuracy, was detected in all eight phenotyping datasets. The type of phenotypic data in which QTL were detected did not have a significant impact on the effect of the QTL as expressed by their $R^{2}$ value. The average $R^{2}$ value for QTL from lesion-length and rAUDPC sets was $13 \%$ in T63DH and $10 \%$ in T54DH, whereas the average $R^{2}$ for QTL detected in binomial or polynomial sets derived from lesion length or plant mortality were 12 and $10 \%$, respectively.

To distinguish the QTL identified in different methods, specific nomenclature was devoted to each QTL (Fig. 3). QTL SR63.A2.1 was associated with stem resistance to S. sclerotiorum in population T63DH and it was located on chromosome A02 (chrA02); sequential numbers were placed if more than one QTL was identified on the same chromosome. QTL SR63.A2.1 had a negative additive effect ranging between -0.18 and -0.58 ; it was detected using lesion length, lesion-polynomial, and the three rAUDPCs (Supplementary Table S4); this QTL contributed to explain between 11 and $15 \%$ of the phenotypic variation $\left(R^{2}\right)$, with higher $R^{2}$ values where lesion size and rAUDPC datasets were used (Table 3). QTL SR63.A2.2 was detected using lesion length, the three rAUDPCs derived from it, and the lesion-binary sets; it had negative additive effect ranging from -0.12 to -0.65 and contributed to explain between 10 and $17 \%$ of the phenotypic variation. All QTL detected in the mortality-binary set had negative additive values and explained between 10 and $13 \%$ of the phenotypic variation of lines.

In total, five QTL were detected in population T54DH. QTL SR54.A2.1 was detected in all eight phenotypic datasets (Supplementary Table S4) and had the strongest negative additive effect when present in the lesion-length dataset (Table 4). In that set, its presence explained between 15 and $16 \%$ of the phenotypic variation. However, its $R^{2}$ value in the mortalitybinomial and polynomial sets were, on average, 7 and $11 \%$, respectively. This QTL shared chrA02-7594120 as flanking marker with SR54.A2.2 in T54DH and with QTL SR63.A2.1 in population $\mathrm{T} 63 \mathrm{DH}$, suggesting that it may be significantly close to the locus associated with response to $\mathrm{S}$. sclerotiorum. Its other flanking marker, chrA02-10934422, was shared with QTL SR54.A2.3, which was detected in the rAUDPC1 dataset (Fig. 4; Table 4). All remaining QTL in T54DH explained less than $10 \%$ of phenotypic variation, except SR54.A2.3, which explained $14 \%$ (Table 4).

\section{Prediction accuracy}

The most accurate flanking marker in prediction of the phenotype was chrA02-7594120, which was linked to QTL SR63.A2.1 in T63DH, and to SR54.A2.1 and SR54.A2.2 in 
T54DH. This marker had an average accuracy ranging between 60 and $66 \%$ in both populations and was linked to the QTL identified in imputed and nonimputed data and all eight phenotypic datasets. Two other markers, chrA02-7458423 linked to QTL SR63.A2.2 in T63DH and chrA02-10934422 linked to QTL SR54.A2.1, had prediction accuracies ranging between 59 and $60 \%$ (Table 5).

\section{DISCUSSION}

This study evaluated the impact of several phenotyping data and the role of genotyping data imputation and presence or absence of segregation-distorted markers on the QTL mapping of B. napus DH plants inoculated with $S$. sclerotiorum. In total, 16 QTL located on chromosomes A02, A07, A09, C01, and C03

TABLE 3

\begin{tabular}{|c|c|c|c|c|c|c|c|}
\hline \multicolumn{8}{|c|}{ TABLE 3} \\
\hline \multicolumn{8}{|c|}{ Quantitative trait loci (QTL) identified for T63DH populations using different phenotyping and genotyping analyzing methods } \\
\hline QTL name & $\mathrm{Chr}^{\mathrm{a}}$ & Physical interval (bp) & Analysis $^{\mathrm{b}}$ & Scoring systems ${ }^{\mathrm{c}}$ & $\mathrm{LOD}^{\mathrm{d}}$ & $R^{2}(\%)^{\mathrm{e}}$ & $\operatorname{Add}^{f}$ \\
\hline \multirow[t]{7}{*}{ SR63.A2.1 } & A02 & $6,194,984-7,594,120$ & 1 & Lesion & 3.76 & 14 & -0.57 \\
\hline & & & 1 & Lesion-polynomial & 3.11 & 11 & -0.19 \\
\hline & & & 2 & Lesion-polynomial & 3.02 & 11 & -0.18 \\
\hline & & & 3 & Lesion-polynomial & 3.08 & 11 & -0.19 \\
\hline & & & 1 & rAUDPC1 & 4.09 & 15 & -0.45 \\
\hline & & & 1 & rAUDPC2 & 4.11 & 15 & -0.53 \\
\hline & & & 1 & rAUDPC3 & 4.26 & 15 & -0.46 \\
\hline \multirow[t]{11}{*}{ SR63.A2.2 } & A02 & $7,315,579-7,458,423$ & 2 & Lesion & 4.44 & 14 & -0.63 \\
\hline & & & 3 & Lesion & 4.7 & 15 & -0.65 \\
\hline & & & 1 & Lesion-binomial & 2.74 & 10 & -0.12 \\
\hline & & & 2 & Lesion-binomial & 2.84 & 10 & -0.12 \\
\hline & & & 3 & Lesion-binomial & 2.85 & 10 & -0.12 \\
\hline & & & 2 & rAUDPC1 & 4.91 & 15 & -0.51 \\
\hline & & & 3 & rAUDPC1 & 4.97 & 15 & -0.51 \\
\hline & & & 2 & rAUDPC2 & 4.95 & 16 & -0.59 \\
\hline & & & 3 & rAUDPC2 & 5.10 & 16 & -0.60 \\
\hline & & & 2 & rAUDPC3 & 5.21 & 17 & -0.52 \\
\hline & & & 3 & rAUDPC3 & 5.27 & 16 & -0.53 \\
\hline SR63.A2.3 & A02 & $7,315,579-8,650,073$ & 2 & Mortality-binomial & 3.11 & 10 & -0.14 \\
\hline \multirow[t]{2}{*}{ SR63.A7.1 } & A07 & $5,117,104-6,001,704$ & 1 & Mortality-binomial & 3.08 & 10 & -0.14 \\
\hline & & & 2 & Mortality-binomial & 3.45 & 11 & -0.14 \\
\hline SR63.A7.2 & A07 & $5,082,112-5,840,027$ & 3 & Mortality-binomial & 2.93 & 10 & -0.13 \\
\hline \multirow[t]{4}{*}{ SR63.C1.1 } & $\mathrm{C} 01$ & $18,520,875-26,829,272$ & 1 & Mortality-binomial & 3.88 & 13 & -0.16 \\
\hline & & & 3 & Mortality-binomial & 3.66 & 13 & -0.15 \\
\hline & & & 1 & Mortality-polynomial & 3.44 & 13 & -0.25 \\
\hline & & & 3 & Mortality-polynomial & 3.43 & 13 & -0.25 \\
\hline SR63.C1.2 & $\mathrm{C} 01$ & $22,057,760-24,895,526$ & 2 & Mortality-binomial & 3.45 & 11 & -0.15 \\
\hline \multirow[t]{8}{*}{ SR63.C1.3 } & $\mathrm{C} 01$ & $21,846,849-31,347,615$ & 3 & Lesion & 2.94 & 9 & -0.52 \\
\hline & & & 2 & Lesion-polynomial & 3.14 & 11 & -0.19 \\
\hline & & & 3 & Lesion-polynomial & 3.14 & 12 & -0.19 \\
\hline & & & 2 & rAUDPC1 & 2.85 & 9 & -0.39 \\
\hline & & & 3 & rAUDPC1 & 3.1 & 9 & -0.4 \\
\hline & & & 2 & rAUDPC2 & 2.79 & 8 & -0.45 \\
\hline & & & 3 & rAUDPC2 & 3.04 & 9 & -0.46 \\
\hline & & & 3 & rAUDPC3 & 2.96 & 8 & -0.39 \\
\hline SR63.C1.4 & $\mathrm{C} 01$ & $32,371,151-33,230,435$ & 4 & Lesion & 3.09 & 11 & 0.56 \\
\hline \multirow[t]{8}{*}{ SR63.C1.5 } & $\mathrm{C} 01$ & $21,580,914$-random $3,377,685^{\mathrm{g}}$ & 5 & Lesion & 3.13 & 11 & 0.57 \\
\hline & & & 6 & Lesion & 3.13 & 11 & 0.57 \\
\hline & & & 5 & rAUDPC1 & 3.07 & 11 & 0.41 \\
\hline & & & 6 & rAUDPC1 & 3.07 & 11 & 0.41 \\
\hline & & & 5 & rAUDPC2 & 3.01 & 11 & 0.48 \\
\hline & & & 6 & rAUDPC2 & 3.08 & 11 & 0.48 \\
\hline & & & 5 & rAUDPC3 & 3.04 & 11 & 0.41 \\
\hline & & & 6 & rAUDPC3 & 3.04 & 11 & 0.41 \\
\hline \multirow[t]{2}{*}{ SR63.C1.6 } & $\mathrm{C} 01$ & random3,563,014-33,230,435 & 5 & Mortality-binomial & 3.12 & 11 & 0.16 \\
\hline & & & 6 & Mortality-binomial & 3.12 & 11 & 0.16 \\
\hline
\end{tabular}

\footnotetext{
a Chromosome.

${ }^{\mathrm{b}}$ Genotypic data processes: $1=$ nonimputed data, $\chi^{2}$ test at $\alpha=0.001 ; 2=$ like 1 , with $\alpha=0.0001 ; 3=$ like 1 , with no $\chi^{2}$ test; $4=$ imputed data, $\chi^{2}$ test at $\alpha=0.001 ; 5=$ like 4 , with $\alpha=0.0001$; and $6=$ like 4 , with no $\chi^{2}$ test.

${ }^{\mathrm{c}}$ Lesion $=$ lesion length measured 3, 5, 7, 9, and 11 days postinoculation (dpi); Lesion-binomial, $0=$ resistant (lesions $\leq 3 \mathrm{~cm}, 9$ dpi) and $1=$ susceptible (lesions $>3 \mathrm{~cm}$ ); Lesion-polynomial, $0=$ resistant (lesions $\leq 3 \mathrm{~cm}, 9 \mathrm{dpi}$ ), $1=$ susceptible (lesions $>6 \mathrm{~cm}$ ), and $2=$ intermediate; rAUDPC1 to $-3=$ relative area under the disease progress curve for lesion length with readings between 3 and 11 dpi (1), 5 and 11 dpi (2), and 3, 7, and 11 dpi (3); mortality = percentage dead plants at $11 \mathrm{dpi}$; mortality-binomial, $0=$ resistant (mortality $\leq 20 \%, 11 \mathrm{dpi}$ ) and $1=$ susceptible (mortality $>20 \%$ ); mortalitypolynomial, $0=$ resistant (mortality $\leq 20 \%, 11 \mathrm{dpi}$ ), 1 susceptible (mortality $>60 \%$ ), and $2=$ intermediate.

d Final logarithm of odds (LOD) of 4 was used to construct the linkage groups.

e Percentage of phenotypic variation explained by the QTL.

${ }^{\mathrm{f}}$ Additive effect.

${ }^{\mathrm{g}}$ Random $=$ random position on the chromosome.
} 
were identified. Multiple QTL detected on A02 were located between the physical positions of 6,194,984 and 10,934,422 bp. This region seems to be rich in QTL associated with reaction to $S$. sclerotiorum because an additional four QTL have been reported recently by other researchers in the same physical interval; that is, SRA2a, SRA2b, and SRA2c by Qasim et al. (2020) and qSR10-1 by Wei et al. (2014). Similarly, two QTL detected on A09 $(26,006,097$ to $31,321,068 \mathrm{bp})$ also were detected in other studies; for example, QTL SRA9a, SRA9b (Qasim et al. 2020), qSR11-1 (Wei et al. 2014), and SRA9-1 (Wu et al. 2013). Qasim et al. (2020) reported QTL SRC3a and SRC3b in the same physical region of QTL SR54.C.3.1, which was located on chrC03 $(23,485,864$ to $31,673,721 \mathrm{bp})$. Furthermore, in a GWAS study conducted by Gyawali et al. (2016), an SSR marker located on chrC01 (C01-29218479) was associated with SRR resistance; this locus is located within the C01 range as QTL SR63.C1.3 $(21,846,849$ to $31,347,615 \mathrm{bp})$ identified in our study. To our knowledge, QTL SR.63.A7.1 and SR63.A7.2, located on chrA07 $(5,117,104$ to $6,001,704 \mathrm{bp})$, have not been reported previously.

Use of multiple lesion-length readings contributed to the detection of additional QTL compared with the use of a single reading. The use of a single observation of lesion length, typically recorded between 3 and $9 \mathrm{dpi}$, is common in QTL mapping studies conducted in canola to identify genomic regions associated with response to S. sclerotiorum (Derbyshire and Denton-Giles 2016; Qasim et al. 2020; Wu et al. 2013, 2019; Yin et al. 2010; Zhao et al. 2006). In this study, lesion-length data collected at 9 dpi were selected for analysis because they showed the clearest separation between lines before plant mortality significantly increased the number of missing data points. This dataset allowed for the detection of seven QTL. Analyses of rAUDPC1, calculated using five reading times, allowed for the detection of eight QTL, including six of the seven detected using the single reading set. Reducing reading

TABLE 4

Quantitative trait loci (QTL) identified for T54DH populations using different phenotyping and genotyping analyzing methods

\begin{tabular}{|c|c|c|c|c|c|c|c|}
\hline QTL name & $\mathrm{Chr}^{\mathrm{a}}$ & Interval & Analysis $^{\mathrm{b}}$ & Scoring systems ${ }^{\mathrm{c}}$ & $\mathrm{LOD}^{\mathrm{d}}$ & $R^{2}(\%)^{\mathrm{e}}$ & $\operatorname{Add}^{f}$ \\
\hline \multirow[t]{20}{*}{ SR54.A2.1 } & \multirow[t]{20}{*}{$\mathrm{A} 02$} & \multirow[t]{20}{*}{$7,594,120-10,934,422$} & 4 & Lesion & 8.33 & 16 & -0.93 \\
\hline & & & 5 & Lesion & 8.33 & 16 & -0.93 \\
\hline & & & 6 & Lesion & 8.04 & 15 & -0.88 \\
\hline & & & 5 & Lesion-binomial & 3.19 & 7 & -0.10 \\
\hline & & & 4 & Lesion-polynomial & 5.83 & 13 & -0.26 \\
\hline & & & 5 & Lesion-polynomial & 5.83 & 13 & -0.26 \\
\hline & & & 6 & Lesion-polynomial & 5.54 & 13 & -0.25 \\
\hline & & & 4 & Mortality-binomial & 2.73 & 6 & -0.12 \\
\hline & & & 5 & Mortality-binomial & 3.57 & 8 & -0.15 \\
\hline & & & 5 & Mortality-polynomial & 4.96 & 11 & -0.24 \\
\hline & & & 6 & Mortality-polynomial & 4.47 & 11 & -0.22 \\
\hline & & & 4 & rAUDPC1 & 7.19 & 14 & -0.52 \\
\hline & & & 6 & rAUDPC1 & 8.13 & 14 & -0.52 \\
\hline & & & 6 & rAUDPC1 & 8.13 & 14 & -0.52 \\
\hline & & & 4 & rAUDPC2 & 7.42 & 14 & -0.63 \\
\hline & & & 5 & rAUDPC2 & 7.42 & 14 & -0.63 \\
\hline & & & 6 & rAUDPC2 & 8.39 & 14 & -0.64 \\
\hline & & & 4 & rAUDPC3 & 6.94 & 13 & -0.48 \\
\hline & & & 5 & rAUDPC3 & 6.94 & 13 & -0.48 \\
\hline & & & 6 & rAUDPC3 & 7.82 & 13 & -0.48 \\
\hline SR54.A2.2 & A02 & $6,194,984-7,594,120$ & 4 & Lesion-binomial & 3.21 & 7 & -0.10 \\
\hline SR54.A2.3 & A02 & $7,315,716-10,934,422$ & 5 & rAUDPC1 & 7.21 & 14 & -0.54 \\
\hline \multirow[t]{2}{*}{ SR54.A9.1 } & \multirow[t]{2}{*}{ A09 } & \multirow[t]{2}{*}{ random $2,813,928-26,006,097^{\mathrm{g}}$} & 6 & rAUDPC1 & 3.71 & 6 & 0.34 \\
\hline & & & 6 & rAUDPC2 & 3.79 & 6 & 0.41 \\
\hline \multirow[t]{11}{*}{ SR54.C3.1 } & \multirow[t]{11}{*}{$\mathrm{C} 03$} & \multirow[t]{11}{*}{$23,485,864-31,673,721$} & 4 & Lesion & 3.60 & 6 & -0.59 \\
\hline & & & 5 & Lesion & 3.56 & 6 & -0.58 \\
\hline & & & 4 & rAUDPC1 & 3.90 & 7 & -0.36 \\
\hline & & & 5 & rAUDPC1 & 3.88 & 6 & -0.36 \\
\hline & & & 6 & rAUDPC1 & 4.58 & 7 & -0.38 \\
\hline & & & 4 & rAUDPC2 & 3.94 & 7 & -0.44 \\
\hline & & & 5 & rAUDPC2 & 3.91 & 7 & -0.44 \\
\hline & & & 6 & rAUDPC2 & 4.63 & 7 & -0.46 \\
\hline & & & 4 & rAUDPC3 & 3.71 & 6 & -0.34 \\
\hline & & & 5 & rAUDPC 3 & 3.68 & 6 & -0.33 \\
\hline & & & 6 & rAUDPC3 & 4.34 & 7 & -0.35 \\
\hline
\end{tabular}

\footnotetext{
${ }^{a}$ Chromosome.

${ }^{\mathrm{b}}$ Genotypic data processes: $1=$ nonimputed data, $\chi^{2}$ test at $\alpha=0.001 ; 2=$ like 1 , with $\alpha=0.0001 ; 3=$ like 1 , with no $\chi^{2}$ test; $4=$ imputed data, $\chi^{2}$ test at $\alpha=0.001 ; 5=$ like 4 , with $\alpha=0.0001$; and $6=$ like 4 , with no $\chi^{2}$ test.

${ }^{\mathrm{c}}$ Lesion $=$ lesion length measured 3, 5, 7, 9, and 11 days postinoculation (dpi); Lesion-binomial, $0=$ resistant (lesions $\leq 3 \mathrm{~cm}, 9$ dpi) and $1=$ susceptible (lesions $>3 \mathrm{~cm}$ ); Lesion-polynomial, $0=$ resistant (lesions $\leq 3 \mathrm{~cm}, 9 \mathrm{dpi}$ ), $1=$ susceptible (lesions $>6 \mathrm{~cm}$ ), and $2=$ intermediate; rAUDPC1 to $-3=$ relative area under the disease progress curve for lesion length with readings between 3 and 11 dpi (1), 5 and 11 dpi (2), and 3, 7, and 11 dpi (3); mortality = percentage dead plants at $11 \mathrm{dpi}$; mortality-binomial, $0=$ resistant (mortality $\leq 20 \%, 11 \mathrm{dpi}$ ) and $1=$ susceptible (mortality $>20 \%$ ); mortality-polynomial, $0=$ resistant (mortality $\leq 20 \%, 11 \mathrm{dpi}$ ), 1 susceptible (mortality $>60 \%$ ), and $2=$ intermediate.

${ }^{\mathrm{d}}$ Final logarithm of odds (LOD) of 4 was used to construct the linkage groups.

e Percentage of phenotypic variation explained by the QTL.

${ }^{\mathrm{f}}$ Additive effect.

${ }^{\mathrm{g}}$ Random $=$ random position on the chromosome.
} 
times to four and three (rAUDPC2 and rAUDPC3) resulted in identification of seven and six QTL, respectively.

Deriving binomial and polynomial sets from a single lesionlength reading was beneficial to the outcome of the study. Although the analysis of the derived sets detected fewer QTL in both populations, it allowed for the identification of QTL SR54.A2.2 in T54DH, which was not detected in the lesionlength data set. QTL SR54.A2.2 was in the same physical location in the reference genome as SR63.A2.1, suggesting that they are the same. SR63.A2.1 and SR63.A2.2 had the highest selection efficiency. In addition, the average $R^{2}$ values of QTL detected in continuous data sets ranged between 10.3 and $12.3 \%$ in T54DH and T63DH, respectively, while those detected using binomial and polynomial sets ranged between 9.8 and $11.2 \%$ for the two populations, respectively. Results of this study demonstrate that analyzing categorical data derived from these continuous data sets may complement and enhance the outcome.

When used alone, continuous phenotyping datasets (i.e., lesion size and rAUDPC) were more reliable for QTL mapping analysis than categorical datasets in this pathosystem. This finding contrasts with those reported by researchers conducting GWAS on other pathosystems (Oladzad et al. 2019; Zitnick-Anderson et al. 2020). It is possible that this discrepancy was due, in part, to differences in the magnitude of the effect of each QTL. Large-effect QTL may approach the qualitative response status that is more easily detected when using categorical datasets while QTL with smaller effect may be masked when the data set is converted into categorical data. In addition, biparental mapping populations are genetically less diverse than those used in GWAS. Reducing the number of phenotypic categories in the former population contributes to the reduced probability of detecting QTL.

The role of plant mortality (percentage of the dead plants) as a scoring system also was evaluated in this study. Distribution of this dataset was skewed to the right and showed that, at $11 \mathrm{dpi}$, many lines had live plants with large lesions (susceptible reaction). Recording mortality percentage a few days later (e.g., $15 \mathrm{dpi}$ ) could solve this issue because distribution of mortality was shifting toward normality as the distance between inoculation and mortality measurement increased. The benefit of conducting QTL analysis on binomial and polynomial data sets derived from plant mortality may have been affected by the magnitude of the mortality observed in the populations. In $\mathrm{T} 63 \mathrm{DH}$, which had an average plant mortality of $41 \%$, it contributed six QTL that had not been detected using continuous data sets. In contrast, in T54DH, which had an average mortality $<10 \%$, it allowed detection of a single marker, SR54.A2.1, which also was detected in continuous data sets.

GBS is a cost-effective and efficient method in development of high-density genetic maps; however, it normally generates a high level of missing data (Elshire et al. 2011; Rutkoski et al. 2013). Genomic imputation can recover a high percentage of this missing information (Huang et al. 2014). Although imputation of
Non-imputed data

$\mathrm{A} 02$

A07

C01

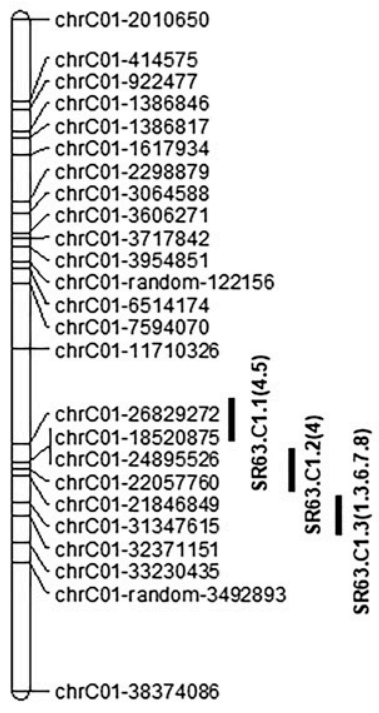

Imputed data

c01

FIGURE 3

Partial genetic linkage map and significant quantitative trait loci (QTL) identified in Brassica napus T63DH population using different phe-

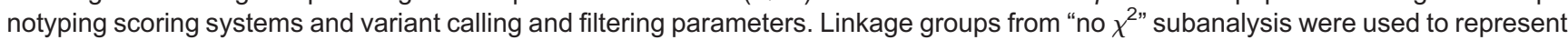
QTL identified in all subanalyses of nonimputed and imputed datasets. Vertical bars show the flanking markers of the QTL. QTL in red have overlap with the physical position of another QTL. Complete linkage groups and QTL information are available in Supplementary Table S3. Name of the QTL consists of the initials of the trait, name of the resistant parent in the mapping population, and chromosome (chr) number; a number was added if more than one QTL were identified on the same chromosome. For each QTL, numbers in parentheses represent phenotyping scoring systems used in QTL detection: 1 = lesion length; 2 = lesion length-binomial; $3=$ lesion length polynomial; 4 = mortality-binomial; 5 = mortality-polynomial; 6 = relative area under the disease progress curve 1 (rAUDPC1), calculated using readings between 3 and 11 days postinoculation (dpi); 7 = rAUDPC2, calculated using readings between 5 and 11 dpi; and $8=$ rAUDPC3, calculated using readings from 3,7 , and $11 \mathrm{dpi}$. 
the genotyping data are not a common step in QTL biparental mapping studies due to the categorical nature of the data (Demetrashvili et al. 2013), application of genotyping data imputation has been reported in some biparental QTL analyses studies. In a study conducted by Spindel et al. (2013), imputation of the genetic data expanded the genetic map of a recombinant inbred line population of rice. Our results agree with their findings because data imputation increased the number of markers available for mapping and the total map length of B. napus by 41 and $56 \%$, respectively, in the T63DH population and by 72 and $53 \%$, respectively, in the T54DH population. Although data imputation increased the number of markers significantly, that number could have been even higher by using paired-end GBS analysis (Clevenger et al. (2015).

The effect of data imputation on the number of QTL detected was population dependent. Fewer genomic regions associate with SSR were identified in $\mathrm{T} 63 \mathrm{DH}$ where data were imputed; this could be due to inserting extra markers between the flaking markers of the detected QTL and, therefore, masking those QTL. In T54DH, application of data imputation was essential because the high level of missing data hindered identification of significant QTL in nonimputed sets. Both imputed and nonimputed data could identify QTL that are located in the genomic regions known to be involved in reactions to SSR (Gyawali et al. 2016; Qasim et al. 2020; Wei et al. 2014; Wu et al. 2013), which verifies the accuracy of these methods in the QTL analysis of biparental populations.

Presence of segregation-distorted regions in $\mathrm{DH}$ populations, specifically in microspore culture-derived populations, has been verified (Alheit et al. 2011). Although some researchers have found the exclusion of distorted markers useful (ArifUzZaman et al. 2016; Huang and Yan 2019; Pascual et al. 2016), others considered that it excluded too many unique markers (Tyrka et al. 2018). Furthermore, it has been theorized that segregation

\section{Imputed data}

\begin{tabular}{lll}
\hline $\mathrm{C02}$ & $\mathrm{A09}$
\end{tabular}
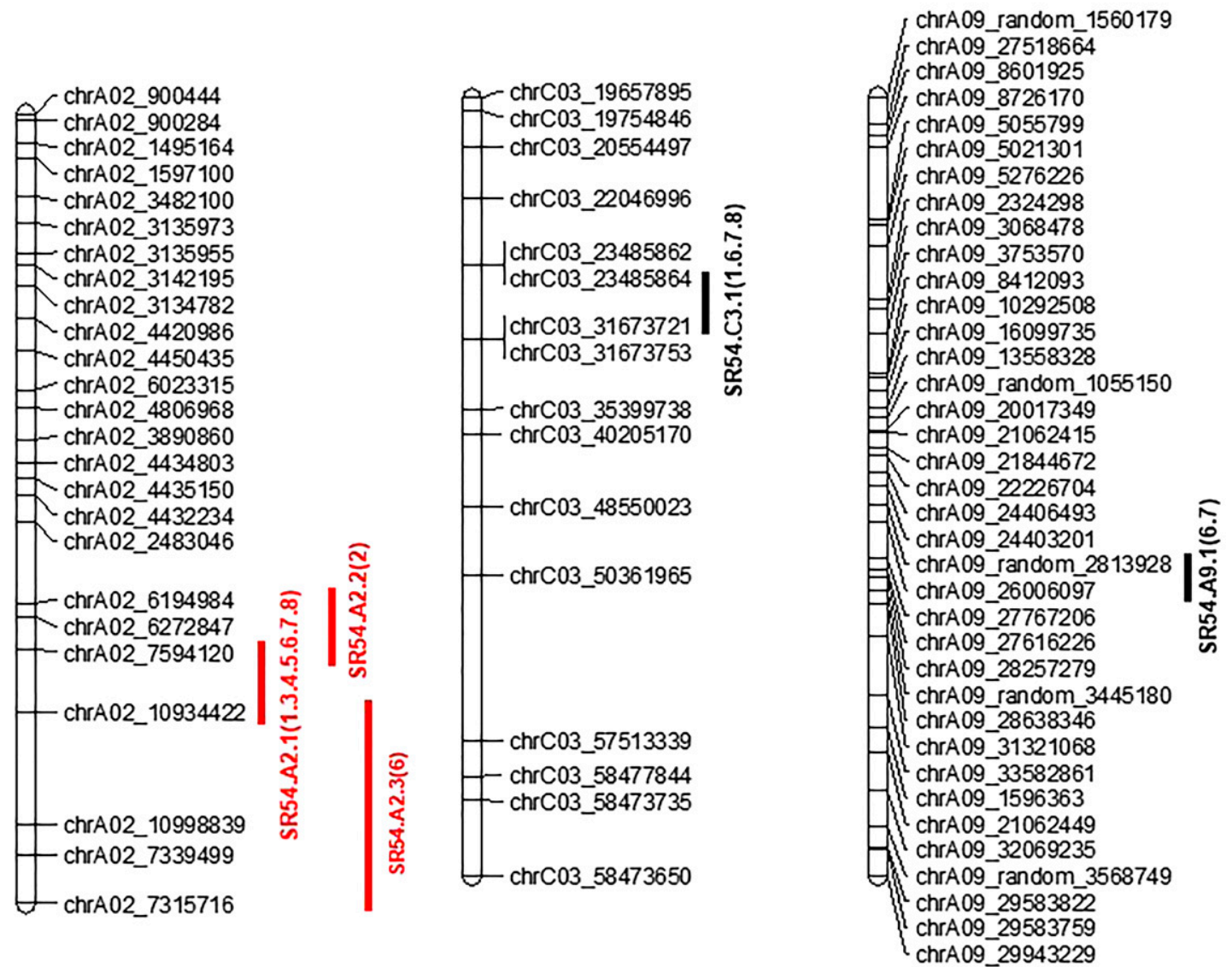

FIGURE 4

Partial genetic linkage maps and significant quantitative trait loci (QTL) identified in Brassica napus T54DH population using different

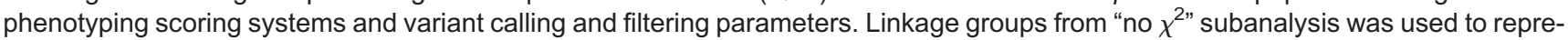
sent QTL identified in all subanalyses of nonimputed and imputed datasets. Vertical bars show the flanking markers of the QTL. QTL in red have overlap with the physical position of another QTL. Complete linkage groups and QTL information are available in Supplementary Table S3. Name of the QTL consists of the initials of the trait, name of the resistant parent in the mapping population, and chromosome (chr) number; a number was added if more than one QTL were identified on the same chromosome. For each QTL, numbers in parentheses represent phenotyping scoring systems that detected the QTL: $1=$ lesion; $2=$ lesion-binomial; $3=$ lesion polynomial; $4=$ mortality-binomial; 5 = mortality-polynomial; 6 = relative area under the disease progress curve 1 (rAUDPC1), calculated using readings between 3 and 11 days postinoculation (dpi); 7 = rAUDPC2, calculated using readings between 5 and 11 dpi; and $8=$ rAUDPC3, calculated using readings from 3,7 , and $11 \mathrm{dpi}$. 
TABLE 5

Prediction accuracy of single nucleotide polymorphism flanking markers linked to quantitative trait loci (QTL) associated with reaction of Brassica napus lines in two doubled haploid mapping populations inoculated with Sclerotinia sclerotiorum

\begin{tabular}{ccc}
\hline Population, QTL & Flanking markers & Prediction accuracy (\%) \\
\hline T63DH & chrA02-6194984 & 56 \\
SR63.A2.1 & chrA02-7594120 & 60 \\
SR63.A2.2 & chrA02-7458423 & 60 \\
& chrA02-7315579 & 45 \\
SR63.A7.1 & chrA07-5117104 & 52 \\
& chrA07-6001704 & 51 \\
SR63.C1.1 & chrC01-26829272 & 42 \\
& chrC01-18520875 & 41 \\
SR63.C1.3 & chrC01-21846849 & 39 \\
& chrC01-31347615 & 45 \\
SR63.C1.5 & chrC01-21580914 & $-{ }^{a}$ \\
& chrC01-random-3377685 & 30 \\
T54DH & & \\
SR54.A2.1 & chrA02-7594120 & 66 \\
SR54.A9.1 & chrA02-10934422 & 58 \\
SR54.C3.1 & chrA09-26006097 & 55 \\
& chrC03-23485864 & 49 \\
& chrC03-31673721 & 56 \\
\hline
\end{tabular}

${ }^{a}$ Markers removed from the genotyping analysis during filtering steps.

distortion has an adverse effect on the power of QTL with dominance effects while it is not necessarily detrimental to those with additive effects (Xu 2008). In DH and recombinant inbred lines, the QTL effects are mainly additive (Gallais 1990; Liu 1997); therefore, including distorted markers in the QTL mapping could be beneficial. Results of our study showed that the number of markers on linkage groups and total genetic map coverage increased when a less stringent $\chi^{2}$ test or no test was used; this agrees with finding of the study conducted by Tyrka et al. (2018). However, the effect of the test on the number of detected QTL and $R^{2}$ values was minimal.

Overall, findings of this study provided insights into the role of phenotyping scoring systems and genotyping analyses in the result of the QTL mapping of a complicated pathosystem where the crop is a polyploid and the nature of the resistance to the disease is quantitative. Using imputed and nonimputed genotyping data and different phenotyping systems resulted in identification of QTL located in regions known to be involved in SSR resistance and in new genomic regions. Markers associated with these regions could be useful in breeding programs after complementary experiments for evaluation of prediction accuracy of markers under the field conditions. Furthermore, using a published B. napus wholegenome sequence (Genoscope website), a list of reported annotated genes associated with the disease defense response which were located between the flanking markers of some QTL identified in this study was prepared (Supplementary Table S5). This list is not intended to be comprehensive because the identification of candidate genes would require a much denser genetic map than the ones used in this study. Nevertheless, the information in this table could be useful to other researchers and for future studies.

\section{ACKNOWLEDGMENTS}

We thank P. E. McClean, Department of Plant Sciences at North Dakota State University (NDSU), and S. Mamidi, HudsonAlpha
Institute for Biotechnology, for their suggestions in genotyping analyses; and Z. Liu, Department of Plant Pathology at NDSU, for reviewing the manuscript and providing valuable suggestions.

\section{LITERATURE CITED}

Alheit, K. V., Reif, J. C., Maurer, H. P., Hahn, V., Weissmann, E. A., Miedaner, T., and Würschum, T. 2011. Detection of segregation distortion loci in triticale ( $x$ Triticosecale Wittmack) based on a highdensity DArT marker consensus genetic linkage map. BMC Genomics 12:380-392. https://doi.org/10.1186/1471-2164-12-380

Amosova, A. V., Zoshchuk, S. A., Volovik, V. T., Shirokova, A. V., Horuzhiy, N. E., Mozgova, G. V., Yurkevich, O. Y., Artyukhova, M. A., Lemesh, V. A., Samatadze, T. E., and Muravenko, O. V. 2019. Phenotypic, biochemical and genomic variability in generations of the rapeseed (Brassica napus L.) mutant lines obtained via chemical mutagenesis. PLoS One 14:e0221699. https://doi.org/10.1371/journal. pone.0221699

Arahana, V. S., Graef, G. L., Specht, J. E., Steadman, J. R., and Eskridge, K. M. 2001. Identification of QTLs for resistance to Sclerotinia sclerotiorum in soybean. Crop Sci. 41:180-188. https://doi.org/10.2135/ cropsci2001.411180x

ArifUzZaman, M., Mamidi, S., McClean, P., and Rahman, M. 2016. QTL mapping for root vigor and days to flowering in Brassica napus $\mathrm{L}$. Can. J. Plant Sci. 97:99-109. https://doi.org/10.1139/cjps-2016-0048

Arkwazee, H. A. 2018. Quantitative trait loci and genome wide association study for resistance to white mold in common (snap) bean. Ph.D. dissertation, Oregon State University.

Chalhoub, B., Denoeud, F., Liu, S., Parkin, I. A., Tang, H., Wang, X., Chiquet, J., Belcram, H., Tong, C., Samans, B., and Corréa, M. 2014. Early allopolyploid evolution in the post-Neolithic Brassica napus oilseed genome. Science 345:950-953. https://doi.org/10.1126/science.1253435

Clevenger, J., Chavarro, C., Pearl, S. A., Ozias-Akins, P., and Jackson, S. A. 2015. Single nucleotide polymorphism identification in polyploids: A review, example, and recommendations. Mol. Plant 8:831-846. https:// doi.org/10.1016/j.molp.2015.02.002

Dai, B., Guo, H., Huang, C., Ahmed, M. M., and Lin, Z. 2017. Identification and characterization of segregation distortion loci on cotton chromosome 18. Front. Plant Sci. 7:2037. https://doi.org/10.3389/fpls.2016.02037

da Silva, C. L., Fritz, A., Clinesmith, M., Poland, J., Dowell, F., and Peiris, K. 2019. QTL mapping of Fusarium head blight resistance and deoxynivalenol accumulation in the Kansas wheat variety 'Everest'. Mol. Breed. 39:35-56. https://doi.org/10.1007/s11032-019-0937-z

Delourme, R., Falentin, C., Fomeju, B. F., Boillot, M., Lassalle, G., André, I., Duarte, J., Gauthier, V., Lucante, N., Marty, A., and Pauchon, M. 2013. High-density SNP-based genetic map development and linkage disequilibrium assessment in Brassica napus L. BMC Genomics 14:120. https://doi.org/10.1186/1471-2164-14-120

del Río, L. E., Bradley, C. A., Henson, R. A., Endres, G. J., Hanson, B. K., McKay, K., Halvorson, M., Porter, P. M., Le Gare, D. G., and Lamey, H. A. 2007. Impact of Sclerotinia stem rot on yield of canola. Plant Dis. 91:191-194. https://doi.org/10.1094/PDIS-91-2-0191

Demetrashvili, N., Van den Heuvel, E. R., and Wit, E. C. 2013. Probability genotype imputation method and integrated weighted lasso for QTL identification. BMC Genet. 14:125. https://doi.org/10.1186/1471-2156-14-125

Derbyshire, M. C., and Denton-Giles, M. 2016. The control of sclerotinia stem rot on oilseed rape (Brassica napus): Current practices and future opportunities. Plant Pathol. 65:859-877. https://doi.org/10.1111/ppa.12517

Disi, J. O., Mei, J., Wei, D., Ding, Y., and Qian, W. 2014. Inheritance of leaf and stem resistance to Sclerotinia sclerotiorum in a cross between Brassica incana and Brassica oleracea var. alboglabra. J. Agric. Sci. 152:146-152. https://doi.org/10.1017/S0021859613000087

Elshire, R. J., Glaubitz, J. C., Sun, Q., Poland, J. A., Kawamoto, K., Buckler, E. S., and Mitchell, S. E. 2011. A robust, simple genotypingby-sequencing (GBS) approach for high diversity species. PLoS One 6:e19379. https://doi.org/10.1371/journal.pone.0019379

Ferrie, A. M. R., Dirpaul, J., Krishna, P., and Keller, W. A. 2005. Effects of brassinosteroids on microspore embryogenesis in Brassica species. In Vitro Cell. Dev. Biol. 41:742-745. https://doi.org/10. 1079/IVP2005690 
Fragoso, C. A., Heffelfinger, C., Zhao, H., and Dellaporta, S. L. 2016. Imputing genotypes in biallelic populations from low-coverage sequence data. Genetics 202:487-495. https://doi.org/10.1534/genetics. 115.182071

Gallais, A. 1990. Quantitative genetics of doubled haploid populations and application to the theory of line development. Genetics 124:199-206. https://doi.org/10.1093/genetics/124.1.199

Gamborg, O. L., Miller, R., and Ojima, K. 1968. Nutrient requirements of suspension cultures of soybean root cells. Exp. Cell Res. 50:151-158. https://doi.org/10.1016/0014-4827(68)90403-5

Garg, H., Li, H., Sivasithamparam, K., and Barbetti, M. J. 2013. Differentially expressed proteins and associated histological and disease progression changes in cotyledon tissue of a resistant and susceptible genotype of Brassica napus infected with Sclerotinia sclerotiorum. PLoS One 8:e65205. https://doi.org/10.1371/journal.pone.0065205

Girke, A., Schierholt, A., and Becker, H. C. 2012. Extending the rapeseed gene pool with resynthesized Brassica napus II: Heterosis. Theor. Appl. Genet. 124:1017-1026. https://doi.org/10.1007/s00122-0111765-7

Gyawali, S., Harrington, M., Durkin, J., Horner, K., Parkin, I. A., Hegedus, D. D., Bekkaoui, D., and Buchwaldt, L. 2016. Microsatellite markers used for genome-wide association mapping of partial resistance to Sclerotinia sclerotiorum in a world collection of Brassica napus. Mol. Breed. 36:72-84. https://doi.org/10.1007/s1 1032-016-0496-5

Howell, E. C., Kearsey, M. J., Jones, G. H., King, G. J., and Armstrong, S. J. 2008. A and C genome distinction and chromosome identification in Brassica napus by sequential fluorescence in situ hybridization and genomic in situ hybridization. Genetics 180:1849-1857. https://doi.org/ 10.1534/genetics.108.095893

Huang, B. E., Raghavan, C., Mauleon, R., Broman, K. W., and Leung, H. 2014. Efficient imputation of missing markers in low-coverage genotyping-by-sequencing data from multiparental crosses. Genetics 197:401-404. https://doi.org/10.1534/genetics.113.158014

Huang, L., and Yan, X. 2019. Construction of a genetic linkage map in Pyropia yezoensis (Bangiales, Rhodophyta) and QTL analysis of several economic traits of blades. PLoS One 14:e0209128. https://doi. org/10.1371/journal.pone.0209128

Joshi, N. A., and Fass, J. N. 2011. sickle: A sliding-window, adaptive, quality-based trimming tool for FastQ files (version 1.33). https:// github.com/najoshi/sickle

Koboldt, D. C., Zhang, Q., Larson, D. E., Shen, D., McLellan, M. D., Lin, L., Miller, C. A., Mardis, E. R., Ding, L., and Wilson, R. K. 2012. VarScan 2: Somatic mutation and copy number alteration discovery in cancer by exome sequencing. Genome Res. 22:568-576. https://doi.org/ 10.1101/gr.129684.111

Kosambi, D. D. 1943. The estimation of map distances from recombination values. Ann. Eugen. 12:172-175. https://doi.org/10.1111/j.1469-1809.1943. tb02321.x

Lara, L. A. de C., dos Santos, J. B., Veloso, J. S., Balestre, M., Alves, F. C., and Leite, M. E. 2014. Identification of QTLs for resistance to Sclerotinia sclerotiorum in carioca common bean by the moving away method. Int. Scholarly Res. Not. 2014:828102. https://doi.org/10.1155/2014/828102

Li, H. 2013. Aligning sequence reads, clone sequences and assembly contigs with BWA-MEM. arXiv. https://arxiv.org/abs/1303.3997

Li, H., Handsaker, B., Wysoker, A., Fennell, T., Ruan, J., Homer, N., Marth, G., Abecasis, G., and Durbin, R. 2009. The sequence alignment/ map format and SAMtools. Bioinformatics 25:2078-2079. https://doi. org/10.1093/bioinformatics/btp352

Liu, B. H. 1997. Statistical Genomics: Linkage, Mapping, and QTL Analysis. CRC Press, Boca Raton, FL, U.S.A.

Lorieux, M. 2012. MapDisto: Fast and efficient computation of genetic linkage maps. Mol. Breed. 30:1231-1235. https://doi.org/10.1007/ s11032-012-9706-y

Lu, K., Wei, L., Li, X., Wang, Y., Wu, J., Liu, M., Zhang, C., Chen, Z., Xiao, Z., Jian, H., and Cheng, F. 2019. Whole-genome resequencing reveals Brassica napus origin and genetic loci involved in its improvement. Nat. Commun. 10:1154. https://doi.org/10.1038/s41467019-09134-9

Meng, L., Li, H., Zhang, L., and Wang, J. 2015. QTL IciMapping: Integrated software for genetic linkage map construction and quantitative trait locus mapping in biparental populations. Crop J. 3:269-283. https:// doi.org/10.1016/j.cj.2015.01.001

Miklas, P. N., Porter, L. D., Kelly, J. D., and Myers, J. R. 2013 Characterization of white mold disease avoidance in common bean. Eur. J. Plant Pathol. 135:525-543. https://doi.org/10.1007/s10658-012-0153-8

Mkwaila, W., Terpstra, K. A., Ender, M., and Kelly, J. D. 2011. Identification of QTL for agronomic traits and resistance to white mold in wild and landrace germplasm of common bean. Plant Breed. 130:665-672. https://doi.org/10.1111/j.1439-0523.2011.01876.x

Mohammadi, P. P., Moieni, A., Ebrahimi, A., and Javidfar, F. 2012. Doubled haploid plants following colchicine treatment of microsporederived embryos of oilseed rape (Brassica napus L.). Plant Cell Tissue Org. 108:251-256. https://doi.org/10.1007/s11240-011-0036-2

Nixon, J. 2006. Testing for segregation distortion in genetic scoring data from backcross or doubled haploid populations. Heredity 96:290-297. https://doi.org/10.1038/sj.hdy.6800797

Oladzad, A., Zitnick-Anderson, K., Jain, S., Simons, K., Osorno, J. M., McClean, P. E., and Pasche, J. 2019. Genotypes and genomic regions associated with Rhizoctonia solani resistance in common bean. Front. Plant Sci. 10:956. https://doi.org/10.3389/fpls.2019.00956

Oladzad-Abbasabadi, A., Kumar, A., Pirseyedi, S., Salsman, E., Dobrydina, M., Poudel, R. S., AbuHammad, W. A., Chao, S., Faris, J. D., and Elias, E. M. 2018. Identification and validation of a new source of low grain cadmium accumulation in durum wheat. G3: Genes Genomes Genet. 8:923-932. https://doi.org/10.1534/g3.117.300370

Pascual, L., Albert, E., Sauvage, C., Duangjit, J., Bouchet, J. P., Bitton, F., Desplat, N., Brunel, D., Le Paslier, M. C., Ranc, N., and Bruguier, L. 2016. Dissecting quantitative trait variation in the resequencing era: Complementarity of bi-parental, multi-parental and association panels. Plant Sci. 242:120-130. https://doi.org/10.1016/j.plantsci.2015.06.017

Pérez-Vega, E., Pascual, A., Campa, A., Giraldez, R., Miklas, P. N., and Ferreira, J. J. 2012. Mapping quantitative trait loci conferring partial physiological resistance to white mold in the common bean RIL population Xana $\times$ Cornell 49242. Mol. Breed. 29:31-41. https://doi. org/10.1007/s11032-010-9522-1

Porcu, E., Sanna, S., Fuchsberger, C., and Fritsche, L. G. 2013. Genotype imputation in genome-wide association studies. Curr. Protoc. Hum. Genet. 78:e84. https://doi.org/10.1002/cphg.84

Qasim, M. U., Zhao, Q., Shahid, M., Samad, R. A., Ahmar, S., Wu, J., Fan, C., and Zhou, Y. 2020. Identification of QTLs containing resistance genes for Sclerotinia stem rot in Brassica napus using comparative transcriptomic studies. Front. Plant Sci. 11:776-789. https://doi.org/10.3389/fpls.2020.00776

Rothmann, L. A., and McLaren, N. W. 2018. Sclerotinia sclerotiorum disease prediction: A review and potential applications in South Africa. S. Afr. J. Sci. 114:1-9. https://doi.org/10.17159/sajs.2018/20170155

Rutkoski, J. E., Poland, J., Jannink, J.-L., and Sorrells, M. E. 2013. Imputation of unordered markers and the impact on genomic selection accuracy. G3: Genes Genomes Genet. 3:427-439. https://doi.org/10. $1534 / \mathrm{g} 3.112 .005363$

Saharan, G. S., and Mehta, N. 2008. Sclerotinia Diseases of Crop Plants: Biology, Ecology and Disease Management. Springer Science \& Business Media, Berlin, Heidelberg, Germany. https://doi.org/10.1007/ 978-1-4020-8408-9

Salsman, E., Kumar, A., AbuHammad, W., Abbasabadi, A. O., Dobrydina, M., Chao, S., Li, X., Manthey, F. A., and Elias, E. M. 2018. Development and validation of molecular markers for grain cadmium in durum wheat. Mol. Breed. 38:28-39. https://doi.org/10. 1007/s11032-018-0788-Z

Scheet, P., and Stephens, M. 2006. A fast and flexible statistical model for large-scale population genotype data: Applications to inferring missing genotypes and haplotypic phase. Am. J. Hum. Genet. 78:629644. https://doi.org/10.1086/502802

Shahoveisi, F., and del Río Mendoza, L. E. 2020. Effect of wetness duration and incubation temperature on development of ascosporic infections by Sclerotinia sclerotiorum. Plant Dis. 104:1817-1823. https://doi.org/10.1094/PDIS-06-19-1304-RE

Snowdon, R., Lühs, W., and Friedt, W. 2007. Oilseed Rape. Springer, Berlin, Heidelberg, Germany. https://doi.org/10.1007/978-3-540-34388-2_2

Soule, M., Porter, L., Medina, J., Santana, G. P., Blair, M. W., and Miklas, P. N. 2011. Comparative QTL map for white mold resistance 
in common bean, and characterization of partial resistance in dry bean lines VA19 and I9365-3. Crop Sci. 51:123-139. https://doi.org/10.2135/ cropsci2010.06.0356

Spindel, J., Wright, M., Chen, C., Cobb, J., Gage, J., Harrington, S., Lorieux, M., Ahmadi, N., and McCouch, S. 2013. Bridging the genotyping gap: Using genotyping by sequencing (GBS) to add highdensity SNP markers and new value to traditional bi-parental mapping and breeding populations. Theor. Appl. Genet. 126:2699-2716. https:// doi.org/10.1007/s00122-013-2166-X

Sun, F., Fan, G., Hu, Q., Zhou, Y., Guan, M., Tong, C., Li, J., Du, D., Qi, C., Jiang, L., and Liu, W. 2017. The high-quality genome of Brassica napus cultivar 'ZS 11'reveals the introgression history in semi-winter morphotype. Plant J. 92:452-468. https://doi.org/10.1111/tpj.13669

Swarts, K., Li, H., Romero Navarro, J. A., An, D., Romay, M. C., Hearne, S., Acharya, C., Glaubitz, J. C., Mitchell, S., Elshire, R. J., and Buckler, E. S. 2014. Novel methods to optimize genotypic imputation for low-coverage, next-generation sequence data in crop plants. Plant Genome 7:1-12. https://doi.org/10.3835/plantgenome2014.05.0023

Tyrka, M., Oleszczuk, S., Rabiza-Swider, J., Wos, H., Wedzony, M., Zimny, J., Ponitka, A., Ślusarkiewicz-Jarzina, A., Metzger, R. J., Baenziger, P. S., and Lukaszewski, A. J. 2018. Populations of doubled haploids for genetic mapping in hexaploid winter triticale. Mol. Breed. 38:46-61. https://doi.org/10.1007/s11032-018-0804-3

USDA-NASS. 2019. United States Department of Agriculture National Agricultural Statistics Service. https://www.nass.usda.gov

Vasconcellos, R. C., Oraguzie, O. B., Soler, A., Arkwazee, H., Myers, J. R., Ferreira, J. J., Song, Q., McClean, P., and Miklas, P. N. 2017. Meta-QTL for resistance to white mold in common bean. PLoS One 12:e0171685. https://doi.org/10.1371/journal.pone.0171685

Voorrips, R. E. 2002. MapChart: Software for the graphical presentation of linkage maps and QTLs. J. Hered. 93:77-78. https://doi.org/10.1093/ jhered/93.1.77

Wei, D., Mei, J., Fu, Y., Disi, J. O., Li, J., and Qian, W. 2014. Quantitative trait loci analyses for resistance to Sclerotinia sclerotiorum and flowering time in Brassica napus. Mol. Breed. 34:1797-1804. https://doi.org/10.1007/s11032-014-0139-7

Wu, J., Cai, G., Tu, J., Li, L., Liu, S., Luo, X., Zhou, L., Fan, C., and Zhou, Y. 2013. Identification of QTLs for resistance to Sclerotinia stem rot and BnaC.IGMT5.a as a candidate gene of the major resistant QTL SRC6 in Brassica napus. PLoS One 8:e67740. https://doi.org/10. 1371/journal.pone.0067740
Wu, J., Chen, P., Zhao, Q., Cai, G., Hu, Y., Xiang, Y., Yang, Q., Wang, Y., and Zhou, Y. 2019. Co-location of QTL for Sclerotinia stem rot resistance and flowering time in Brassica napus. Crop J. 7:227-237. https://doi.org/10.1016/j.cj.2018.12.007

Xie, W., Feng, Q., Yu, H., Huang, X., Zhao, Q., Xing, Y., Yu, S., Han, B., and Zhang, Q. 2010. Parent-independent genotyping for constructing an ultrahigh-density linkage map based on population sequencing. Proc. Natl. Acad. Sci. U.S.A. 107:10578-10583. https://doi. org/10.1073/pnas.1005931107

$\mathrm{Xu}$, S. 2008. Quantitative trait locus mapping can benefit from segregation distortion. Genetics 180:2201-2208. https://doi.org/10.1534/ genetics.108.090688

Yin, X., Yi, B., Chen, W., Zhang, W., Tu, J., Fernando, W. D., and Fu, T. 2010. Mapping of QTLs detected in a Brassica napus DH population for resistance to Sclerotinia sclerotiorum in multiple environments. Euphytica 173:25-35. https://doi.org/10.1007/s10681-009-0095-1

Yu, F., Zhang, X., Peng, G., Falk, K. C., Strelkov, S. E., and Gossen, B. D. 2017. Genotyping-by-sequencing reveals three QTL for clubroot resistance to six pathotypes of Plasmodiophora brassicae in Brassica rapa. Sci. Rep. 7:4516. https://doi.org/10.1038/s41598-017-04903-2

Zhao, J., and Meng, J. 2003. Genetic analysis of loci associated with partial resistance to Sclerotinia sclerotiorum in rapeseed (Brassica napus L.). Theor. Appl. Genet. 106:759-764. https://doi.org/10.1007/ s00122-002-1171-2

Zhao, J., Udall, J. A., Quijada, P. A., Grau, C. R., Meng, J., and Osborn, T. C. 2006. Quantitative trait loci for resistance to Sclerotinia sclerotiorum and its association with a homeologous non-reciprocal transposition in Brassica napus L. Theor. Appl. Genet. 112:509-516. https://doi.org/10.1007/s00122-005-0154-5

Zheng, C., Boer, M. P., and van Eeuwijk, F. A. 2018. Accurate genotype imputation in multiparental populations from low-coverage sequence. Genetics 210:71-82. https://doi.org/10.1534/genetics.118.300885

Zitnick-Anderson, K., Oladzadabbasabadi, A., Jain, S., Modderman, C., Osorno, J. M., McClean, P. E., and Pasche, J. S. 2020. Sources of resistance to Fusarium solani and associated genomic regions in common bean diversity panels. Front. Genet. 11:475. https://doi.org/10. 3389/fgene.2020.00475

Zuo, J. F., Niu, Y., Cheng, P., Feng, J. Y., Han, S. F., Zhang, Y. H., Shu, G., Wang, Y., and Zhang, Y. M. 2019. Effect of marker segregation distortion on high density linkage map construction and QTL mapping in Soybean (Glycine max L.). Heredity 123:579-592. https://doi.org/10. 1038/s41437-019-0238-7 